ARTICLE

https://doi.org/10.1038/s41467-019-09181-2

\title{
Calcium-activated 14-3-3 proteins as a molecular switch in salt stress tolerance
}

\author{
Zhijia Yang ${ }^{1}$, Chongwu Wang (D) ${ }^{1}$, Yuan Xue ${ }^{1}$, Xiao Liu', She Chen ${ }^{2}$, ChunPeng Song (D) ${ }^{3}$, Yongqing Yang ${ }^{1} \&$ \\ Yan Guo iD ${ }^{1}$
}

Calcium is a universal secondary messenger that triggers many cellular responses. However, it is unclear how a calcium signal is coordinately decoded by different calcium sensors, which in turn regulate downstream targets to fulfill a specific physiological function. Here we show that SOS2-LIKE PROTEIN KINASE5 (PKS5) can negatively regulate the Salt-Overly-Sensitive signaling pathway in Arabidopsis. PKS5 can interact with and phosphorylate SOS2 at Ser ${ }^{294}$, promote the interaction between SOS2 and 14-3-3 proteins, and repress SOS2 activity. However, salt stress promotes an interaction between 14-3-3 proteins and PKS5, repressing its kinase activity and releasing inhibition of SOS2. We provide evidence that 14-3-3 proteins bind to $\mathrm{Ca}^{2+}$, and that $\mathrm{Ca}^{2+}$ modulates 14-3-3-dependent regulation of SOS2 and PKS5 kinase activity. Our results suggest that a salt-induced calcium signal is decoded by 14-3-3 and SOS3/SCaBP8 proteins, which selectively activate/inactivate the downstream protein kinases SOS2 and PKS5 to regulate $\mathrm{Na}^{+}$homeostasis by coordinately mediating plasma membrane $\mathrm{Na}^{+} / \mathrm{H}^{+}$antiporter and $\mathrm{H}^{+}$-ATPase activity.

\footnotetext{
${ }^{1}$ State Key Laboratory of Plant Physiology and Biochemistry, College of Biological Sciences, China Agricultural University, 100193 Beijing, China. ${ }^{2}$ National Institute of Biological Sciences, Beijing, 7 Science Park Road, Zhongguancun Life Science Park, 102206 Beijing, China. ${ }^{3}$ Collaborative Innovation Center of Crop Stress Biology, Henan Province, Institute of Plant Stress Biology, Henan University, 475001 Kaifeng, China. Correspondence and requests for materials should be addressed to Y.Y. (email: yangyongqing@cau.edu.cn) or to Y.G. (email: guoyan@cau.edu.cn)
} 
C alcium, a universal secondary messenger, is an important regulator of many cellular activities in both plants and animals. Fluctuations in the concentration of cytosolicfree $\mathrm{Ca}^{2+}\left(\left[\mathrm{Ca}^{2+}\right]_{\text {cyt }}\right)$ triggered by internal or external stimuli are decoded by different $\mathrm{Ca}^{2+}$ sensors, such as calmodulin $(\mathrm{CaM})^{1-3}, \mathrm{Ca}^{2+}$-dependent protein kinases $(\mathrm{CDPKs})^{4,5}$, and SOS3-like $\mathrm{Ca}^{2+}$-binding protein/calcineurin $\mathrm{B}$-like protein $(\mathrm{SCaBP} / \mathrm{CBL})^{6-11}$. However, it is unclear how different calcium sensors decode a calcium signal and coordinately regulate the activity of various cellular targets to achieve a specific physiological response.

The salt overly sensitive (SOS) pathway, which is conserved in plants, regulates sodium ion homeostasis under salt stress ${ }^{10,11}$. The major components of the SOS pathway are the SOS3 and SCaBP8 calcium sensors, the SOS2 protein kinase, and the plasma membrane $\mathrm{Na}^{+} / \mathrm{H}^{+}$antiporter SOS1 (PM Na${ }^{+} / \mathrm{H}^{+}$antiporter) ${ }^{12-15}$. Under salt stress, SOS3 and SCaBP8 perceive the salt-induced $\mathrm{Ca}^{2+}$ signal and interact with SOS2, thereby recruiting it to the plasma membrane $e^{14,16,17}$. SOS2 then phosphorylates SOS1 $1^{\text {Ser1138, which }}$ alleviates auto-inhibition of SOS1 by the C-terminal repressor domain, activating SOS1 and increasing $\mathrm{Na}^{+}$efflux ${ }^{18-20}$. Under normal growth conditions (in the absence of salt stress), SOS2 is phosphorylated at $\mathrm{Ser}^{294}$ and interacts with 14-3-3 proteins, which repress the kinase activity of $S O S 2^{21}$. Another protein, GI, also interacts with and represses SOS2 activity under normal growth conditions $^{22}$. However, it is unknown which kinase phosphorylates SOS2 $2^{\mathrm{Ser} 294}$ and how 14-3-3 proteins are regulated to either bind or release SOS2 in the absence or presence of salt stress, respectively.

Activation of the SOS1 $\mathrm{Na}^{+} / \mathrm{H}^{+}$antiporter under salt stress requires that $\mathrm{SOS} 2$ be activated and that a plasma membrane $\mathrm{H}^{+}$ATPase (PM H${ }^{+}$-ATPase)-generated proton gradient be established across the plasma membrane ${ }^{23}$. Activation of the $\mathrm{PM} \mathrm{H}^{+}$ATPase is involved in phosphorylation/dephosphorylation pro-

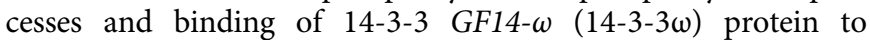
the $\mathrm{PM} \mathrm{H}^{+}$-ATPase AHA2 at $\mathrm{Thr}^{947}$ which relieves its autoinhibition by the C-terminal domain ${ }^{24-28}$. SOS2-LIKE PROTEIN KINASE5 (PKS5) phosphorylates the $\mathrm{PM} \mathrm{H} \mathrm{H}^{+}$-ATPase AHA2 at $\mathrm{Thr}^{931}$ and inhibits its activity by reducing the binding of 14-3$3 \omega$ to $\mathrm{AHA} 2^{\text {Thr947, }}$, which negatively regulates salt-alkaline tolerance of Arabidopsis ${ }^{24}$. Although it is clear that $\mathrm{PM} \mathrm{H}^{+}$-ATPase is activated under salt stress in plant to provide a driving force for the $\mathrm{Na}^{+} / \mathrm{H}^{+}$antiporter, little is known about how these two transporters are coordinately regulated.

In this study, we show that PKS5 can interact with and phosphorylate SOS2. PKS5 can negatively regulate salt tolerance and provide evidence that PKS5 and SOS2 activity is regulated in a $\mathrm{Ca}^{2+}$ - dependent manner. We provide a model whereby $14-$ 3-3 proteins act as a $\mathrm{Ca}^{2+}$-dependent switch to coordinately regulate SOS2 and PKS5, thereby activating both the $\mathrm{PM} \mathrm{Na}{ }^{+} / \mathrm{H}^{+}$ antiporter and $\mathrm{PM} \mathrm{H} \mathrm{H}^{+}$-ATPase and mediating the plant's response to salt stress.

\section{Results}

PKS5 can interact with and phosphorylate SOS2 at Ser294. Phosphorylation of SOS2 2 er294 is important for the regulation of SOS2 kinase activity. To identify the kinase responsible for phosphorylating SOS2 $2^{\mathrm{Ser} 294}$, we obtained Arabidopsis transgenic plants expressing Pro35S:6×Myc-SOS2 in the sos2-2 mutant background ${ }^{17}$ and searched for potential SOS2 interactors by mass spectrometry analysis. Among the list of candidateinteracting proteins, we identified PKS5 (Supplementary Fig. 1a, Supplementary Data 1). We verified the interaction between PKS5 and SOS2 by yeast two-hybrid analysis (Fig. 1a). In addition, a bimolecular fluorescence complementation (BiFC) analysis in Nicotiana benthamiana revealed yellow fluorescent protein
(YFP) fluorescence signal in leaves transiently expressing $Y F P^{N}$ PKS5 and YFP $C_{-S O S 2}$, but not in those expressing YFPN-AHA1 and $Y_{F P} C_{-}$SOS2 (Fig. 1b). Thus, PKS5 can interact with SOS2 in yeast and in plant cells.

We next examined whether PKS5 can phosphorylate SOS2 in vitro. In order to avoid interference caused by autophosphorylation of SOS2, we used a kinase-dead mutant variant $^{12}$, His-tagged $\mathrm{SOS} 2^{\mathrm{K} 40 \mathrm{~N}}$, for the kinase assay, and the result revealed that $\mathrm{SOS} 2^{\mathrm{K} 40 \mathrm{~N}}$ was phosphorylated by PKS5 (Fig. 1c). We also performed an in vitro kinase assay to determine whether SOS2 phosphorylates PKS5, the result showed that SOS2 could not phosphorylate PKS5 ${ }^{\mathrm{K} 50 \mathrm{~N}}$ (a kinase-dead-type mutant) (Supplementary Fig. 1c). To identify the site of SOS2 that is phosphorylated by PKS5, we mapped the SOS2 phosphorylation region. For this, we purified three recombinant glutathione Stransferase (GST)-tagged SOS2-truncated proteins, namely GSTSOS2-KD (the N-terminal kinase domain), GST-SOS2-JD (the junction domain), and GST-SOS2-RD (the regulator domain) ${ }^{21}$. An in vitro phosphorylation assay showed that only the junction domain of SOS2 was phosphorylated by PKS5 (Fig. 1c). Ser ${ }^{294}$ is the only possible phosphorylation site in this peptide ${ }^{21}$ and, as expected, mutation of this residue to Ala abolished the PKS5mediated phosphorylation of $\mathrm{SOS}_{2}{ }^{\mathrm{K} 40 \mathrm{~N}}$ and SOS2-JD. SOS2$\mathrm{JD}^{\mathrm{S} 294 \mathrm{~A}}$ displayed a protein shift compared to the wild-type SOS2-JD peptide (Fig. 1c, d), which suggests that the mutation may change the protein structure or charge. Furthermore, a mass spectrometry assay confirmed that PKS5 phosphorylated SOS2Ser294 (Supplementary Fig. 1b). SCaBP1, a SOS3-like calciumbinding protein, can bind to $\mathrm{Ca}^{2+}$ and activate PKS5 to repress $\mathrm{PM} \mathrm{H}^{+}$-ATPase activity in yeast ${ }^{24}$. We therefore tested whether $\mathrm{Ca}^{2+}$ and SCaBP1 play a role in the phosphorylation of SOS2 by PKS5. In vitro phosphorylation assay showed that $\mathrm{Ca}^{2+}$ and SCaBP1 had no obvious effect on the phosphorylation of SOS2JD by PKS5 (Supplementary Fig. 1d). Overall, these results demonstrate that PKS5 can interact with and phosphorylate SOS2 at Ser294.

PKS5 enhances the interaction between SOS2 and 14-3-3s. As PKS5 can phosphorylate SOS2 ${ }^{\mathrm{Ser} 294}$, we wondered whether PKS5 plays a role in the 14-3-3-mediated repression of SOS2 kinase activity. We first examined whether PKS5 affects the interaction between 14-3-3 proteins and SOS2 in vitro. For this, GST-tagged SOS2-JD, GST-tagged SOS2-JDS294A, His-tagged PKS5, and Histagged 14-3-3 $\lambda$ proteins purified from Escherichia coli were used for an in vitro pull-down assay. We observed that SOS2-JD interacts with 14-3-3 $\lambda$, and that this interaction was enhanced by the addition of PKS5 particularly in the presence of kinase buffer containing ATP (Fig. 2a). However, the enhancement was reduced when the $\mathrm{SOS} 2^{\mathrm{S} 294 \mathrm{~A}}$ mutant was used (Fig. 2a). To determine the effect of PKS5 on the 14-3-3/SOS2 interaction, we crossed Col-0 expressing Pro35S:6×Myc-SOS2 with the PKS5 lossof-function mutant $p k s 5-1^{24}$, and the gain-of-function mutants, pks5-3 and, pks5-4, the latter of which were isolated in the Col er105 background (subsequently referred to as BigM) and have higher PKS5 kinase activity ${ }^{29}$. Using these crossed lines, MycSOS2 was immunoprecipitated with anti-C-Myc agarose and 14-3-3 proteins were detected with anti-14-3-3 antibody in the co-immunoprecipitated complex. Higher 14-3-3 protein signal resulted from SOS2 immunoprecipitation in the $p k s 5-3$ and $p k s 5$ 4 backgrounds than in the BigM control; however, less immunoprecipitated 14-3-3 protein signal was observed in the pks5-1 background than in Col-0 (Fig. 2b). Split-luciferase complementation (LUC) imaging assays in N. benthamiana also indicated that the presence of PKS5 enhanced the interaction

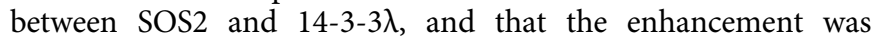


a

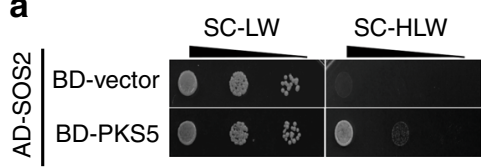

b

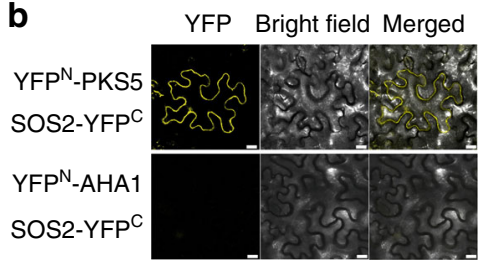

C

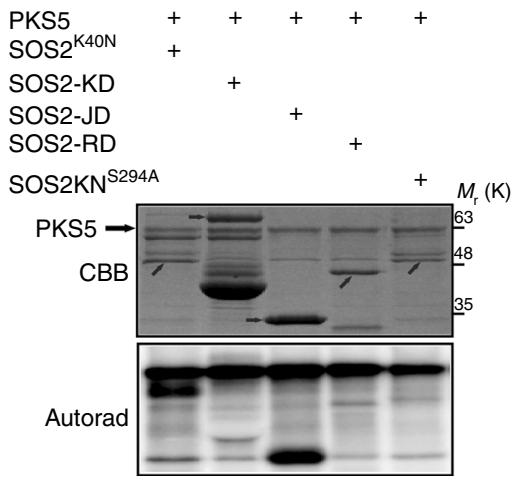

d

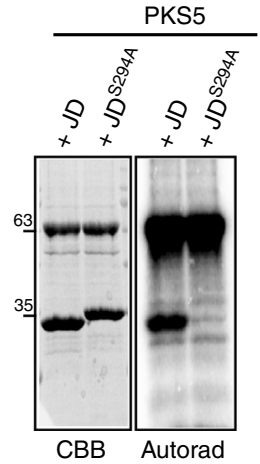

Fig. 1 PKS5 interacts with SOS2 and phosphorylates SOS2 ${ }^{\text {Ser294 }}$ a Yeast two-hybrid assay showing that SOS2 interacts with PKS5. No interaction was observed when using an empty-vector control (BD-vector). b BiFC assay of the SOS2/PKS5 interaction in N. benthamiana. Plasmids containing YFPN-PKS5 and SOS2-YFPC or YFPN-AHA1 and SOS2-YFPC were co-transformed into $N$. benthamiana leaves. Two days post-infiltration, the YFP fluorescence was detected by confocal microscopy. Scale bars, $50 \mu \mathrm{m}$. c In vitro kinase assay showing that PKS5 phosphorylates SOS2 $2^{\mathrm{Ser} 294}$. The recombinant proteins were incubated in kinase buffer at $30^{\circ} \mathrm{C}$ for $30 \mathrm{~min}$ and separated by SDS-PAGE. Arrow indicates the target protein. $\mathbf{d}$ In vitro kinase assay showing that PKS5 phosphorylates SOS2-JD but not SOS2-JDS294A. JD, SOS2-JD; JDS294A, SOS2-JDS294A. CBB Coomassie brilliant blue, Autorad autoradiograph. Source data of $\mathbf{c}$ and $\mathbf{d}$ are provided in the Source Data file

greater when the constitutively active kinases PKS5-3 and PKS5-4 were used (Fig. 2c, d). However, the SOS2 2 294A mutant abolished the PKS5-enhanced interaction between SOS2 and 14-3-3 $\lambda$, and co-expressed $\mathrm{PKS} 5^{\mathrm{K} 50 \mathrm{~N}}$ had no significant effect on this interaction (Supplementary Fig. 2b, c). These results suggest that PKS5 enhances the interaction between SOS2 and 14-3-3 $\lambda$ through phosphorylating the SOS2 $\operatorname{Ser}^{294}$. We also tested whether SCaBP1 has effect on the PKS5-mediated interaction between SOS2 and 14-3-3 $\lambda$, and LUC imaging assays showed that SCaBP1 had no obvious effect on this interaction (Supplementary Fig. 2d, e).

$14-3-3 \lambda$ and $\kappa$ bind to and regulate SOS2 kinase activity ${ }^{21}$. We thus analyzed the effect of PKS5 on SOS2 kinase activity in planta. We first conducted comparative phosphorylation assays using His-tagged SCaBP8 and MBP as substrates for SOS2, and SOS2 immunoprecipitated from transgenic plants expressing Myc-SOS2 in BigM, pks5-3, pks5-4, Col-0, and pks5-1 backgrounds. $\mathrm{NaCl}$ treatment is known to enhance SOS2 kinase activity $^{17}$. The kinase activity of Myc-SOS2 in BigM, pks5-3, and pks5-4 was low before $\mathrm{NaCl}$ treatment, and $\mathrm{NaCl}$-induced activation of SOS2 was repressed in pks5-3 and pks5-4 (Fig. 2e). The base-level kinase activity of SOS2 was increased in pks5-1, and was not further enhanced following $\mathrm{NaCl}$ treatment (Fig. 2f). $\mathrm{SCaBP} 8^{\mathrm{Ser} 237}$ is specifically phosphorylated by $\mathrm{SOS} 2^{17}$, and thus we used anti-phospho-SCaBP8S237 (anti-P-SC8) ${ }^{17}$ to examine SCaBP8 phosphorylation as a measure of SOS2 kinase activity. Total proteins were extracted from transgenic plants expressing $35 S: 6 \times M y c-S C a B P 8$ in the $p k s 5$ and wild type backgrounds with or without $\mathrm{NaCl}$ treatment, and SCaBP8 protein and phosphorylation levels were determined by immunoblot analysis using anti-C-Myc and anti-P-SC8 antibodies, respectively. Consistently, we found that SCaBP8 phosphorylation in pks5-3 and pks5-4 was lower than in the wild type following $\mathrm{NaCl}$ treatment (Fig. $2 \mathrm{~g}$ ), and was higher in $p k s 5-1$ than in the wild type prior to $\mathrm{NaCl}$ treatment (Fig. 2h). These results suggest that PKS5 negatively regulates SOS2 kinase activity by phosphorylating SOS2.

PKS5 inhibits the SOS pathway in yeast through 14-3-3s. To determine the effect of PKS5-mediated SOS2 ${ }^{\mathrm{Ser} 294}$ phosphorylation by PKS5 on the SOS pathway, we performed an experiment in a yeast system named as Sos Recruitment System (SRS) in which the SOS pathway was reconstituted via coexpression of SOS1 and constitutively activated SOS2 $2^{\mathrm{T} 168 \mathrm{D} / \Delta 308}$ in yeast mutants lacking endogenous $\mathrm{Na}^{+}$transporters ${ }^{20}$. We mutated $\mathrm{Ser}^{294}$ of SOS2 to Ala or Asp to generate the phospho-dead or phosphor-mimic status of the protein, respectively. Subsequently, we expressed SOS2 $2^{T 168 D / \Delta 308 / S 294 A}$, SOS2 $2^{T 168 D / \Delta 308 / S 294 D}$, and SOS2 $2^{T 168 D / \Delta 308}$ constructs with or without $14-3-3 \lambda$ in yeast strain Saccharomyces cerevisiae JP837, and assessed the ability of the resulting transformants to grow on Arg phosphate (AP) medium containing $\mathrm{NaCl}$. 14-3-3 $\lambda$ expression did not affect yeast growth on AP medium containing $25 \mathrm{mM} \mathrm{NaCl}$, but limited growth on medium containing $50 \mathrm{mM} \mathrm{NaCl}$ (Supplementary Fig. 2h). There was no growth difference on salt medium when SOS2 $2^{T 168 D / \Delta 308 / S 294 A}$ was expressed compared to $S O S 2^{T 168 D / \Delta 308}$ in the presence or absence of 14-3-3 $\lambda$ (Supplementary Fig. 2f). By contrast, expression of the Ser ${ }^{294}$-to-Asp mutant form (SOS2T168D/A308/S294D), which enhances the 14-3-3 $\lambda /$ SOS2 interaction and represses SOS2 kinase activity ${ }^{21}$, strongly repressed yeast growth on AP medium that contained only $15 \mathrm{mM} \mathrm{NaCl}$ (Supplementary Fig. 2g), and coexpression of 14-3-3 $\lambda$ further increased this repression but had little effect on yeast expressing SOS2 $2^{T 168 D / \Delta 308}$ (Supplementary Fig. 2g).

It is possible that $14-3-3 \lambda$ homologs in yeast repress SOS2 $2168 \mathrm{D} / \triangle 308 / \mathrm{S} 294 \mathrm{D}$ kinase activity. However, the addition of PKS5 failed to repress SOS2 activity in yeast (Supplementary Fig. 2h). Furthermore, the efficient function of PKS5 may require the SOS3-like $\mathrm{Ca}^{2+}$-binding protein $\mathrm{SCaBP} 1^{24}$. As a control, 143-3 $\omega$ did not obviously affect SOS2 activity in the yeast system (Supplementary Fig. 2h). When SOS2 $2^{T 168 D / \Delta 308}$ was coexpressed with 14-3-3i, PKS5-3/PKS5-4 substantially repressed yeast growth, consistent with PKS5 phosphorylation and repression of SOS2T168D/ $\triangle 308$ (Fig. 2i), whereas the coexpression of $14-3-3 \lambda$ and SOS2 $2^{T 168 D / \Delta 308 / S 294 D}$ resulted in no further growth inhibition in the presence of PKS5-3/PKS5-4 (Fig. 2j). Collectively, these results further suggest that PKS5-dependent phosphorylation of SOS2 2 er294 represses SOS2 kinase activity. In yeast this appears to

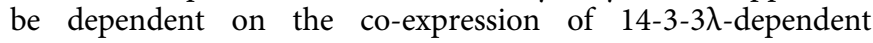
manner.

PKS5 negatively regulates salt tolerance in Arabidopsis. Given our evidence that PKS5 can phosphorylate SOS2 and regulate SOS2 kinase activity, we next determined whether PKS5 affects salt tolerance in Arabidopsis. Following the transfer of 7-day-old seedlings to medium with 75 or $100 \mathrm{mM} \mathrm{NaCl}$, pks5-3 and pks5-4 with enhanced PKS5 activity showed significantly reduced root 
a

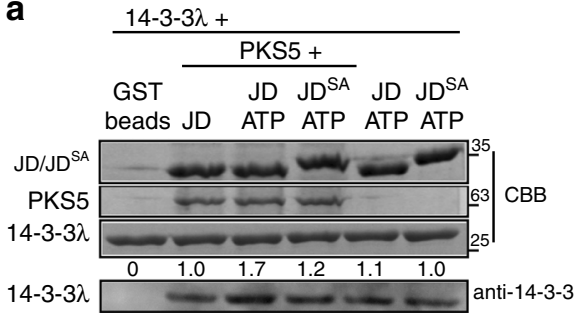

b

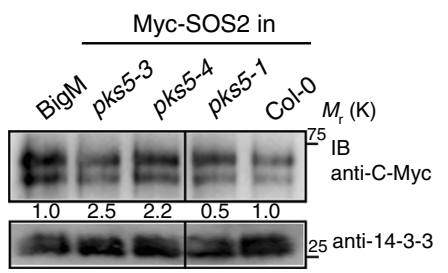

C

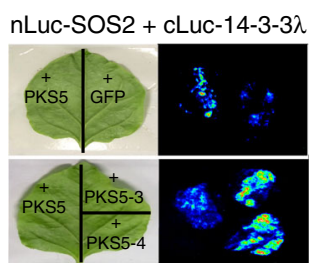

d

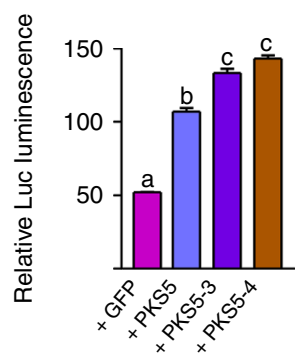

e

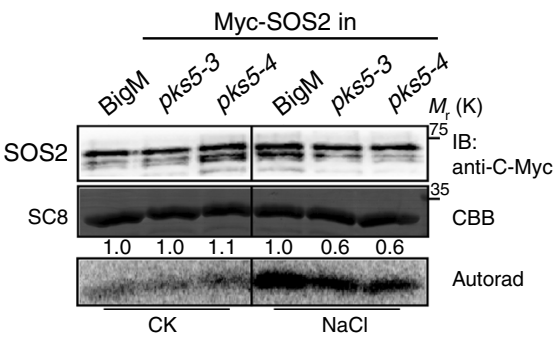

h

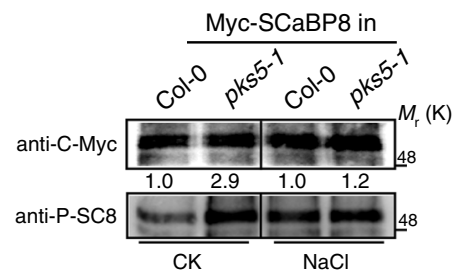

f

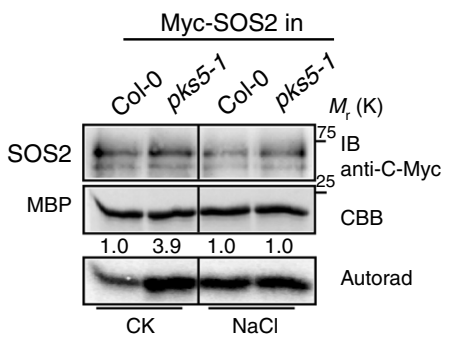

i

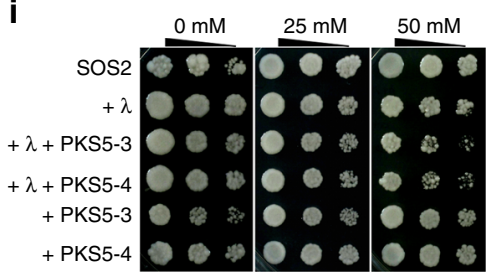

g

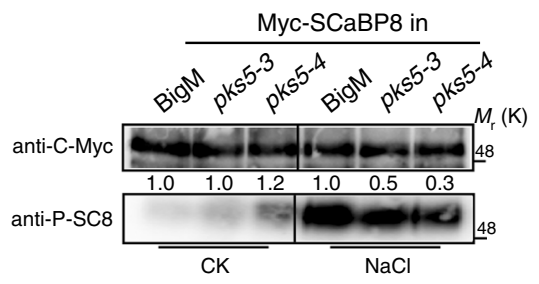

j

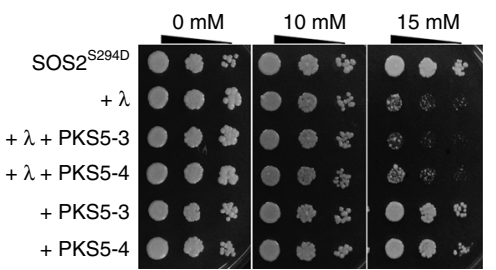

Fig. 2 PKS5 enhances the interaction between SOS2 and 14-3-3 proteins. a In vitro pull-down assay showing that PKS5 enhances the interaction between SOS2-JD and 14-3-3 $\lambda$ following incubation with SOS2-JD in kinase buffer containing ATP. JD, SOS2-JD; JDSA, SOS2-JDS294A. b In vivo Coimmunoprecipitation assay showing that PKS5 enhances the interaction between SOS2 and 14-3-3 proteins. Myc-SOS2 was extracted with anti-C-Myc agarose from 10-day-old seedlings expressing Pro35S:6×Myc-SOS2 in BigM/pks5-3/pks5-4/Col-0/pks5-1 backgrounds and analyzed by immuno blotting with anti-C-Myc and anti-14-3-3 antibodies. c Luciferase complementation imaging assay showing that co-expression of PKS5/PKS5-3/PKS5-4 in N.

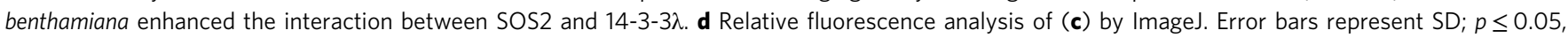
Student's $t$-test; $n=3$; significant differences are indicated by different lowercase letters. e, $\mathbf{f}$ In vitro kinase assay showing that PKS5 negatively regulates SOS2 activity. Myc-SOS2 was extracted using anti-C-Myc agarose from 10-day-old transgenic seedlings treated with or without $100 \mathrm{mM} \mathrm{NaCl}$ for $12 \mathrm{~h}$. His-SCaBP8 (SC8) and MBP were used as the substrates and anti-C-Myc antibody was used for immunoblot analysis. $\mathbf{g}$, $\mathbf{h}$ In vivo Co-immunoprecipitation assay showing that PKS5 negatively regulates the phosphorylation status of SCaBP8. Myc-SCaBP8 was extracted from 10-day-old seedlings expressing Pro35S:6×Myc-SCaBP8 in the BigM/pks5-3/pks5-4/Col-0/pks5-1 backgrounds treated or not with $100 \mathrm{mM} \mathrm{NaCl}$ for $12 \mathrm{~h}$ and analyzed by immunoblotting with anti-C-Myc and anti-phospho-SCaBP8Ser237 (anti-P-SC8) antibodies. $\mathbf{i}, \mathbf{j}$ SOS recruitment system assays showing that PKS5 inhibits the SOS

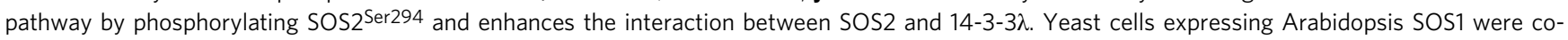
transformed with the indicated plasmids, two positive clones with $3.5 \mu \mathrm{L}$ of serial five-fold dilutions were grown on AP medium with different concentrations of $\mathrm{NaCl}$ to analyze the salt tolerance of yeast strains expressing SOS2, SOS2T168D/ $\Delta 308$; SOS2S294D, SOS2T168D/ $\Delta 308 / S 294 \mathrm{D}$. IB immunoblot. CBB Coomassie Brilliant Blue, Autorad autoradiograph. Source data of $\mathbf{a}, \mathbf{b}$, and $\mathbf{e}-\mathbf{h}$ are provided in the Source Data file

growth compared to the wild type, and the reduction was greater in the presence of $100 \mathrm{mM}$ than $75 \mathrm{mM} \mathrm{NaCl}$ (Fig. 3a, b). However, the root growth of pks5-1 was greater than that of Col-0 when seedlings were transferred to medium containing $125 \mathrm{mM}$ $\mathrm{NaCl}$ (Supplementary Fig. 3a, b). Furthermore, using Noninvasive Micro-test Technology (NMT), we measured $\mathrm{Na}^{+}$flux at the meristem zone of 7-day-old BigM, pks5-3, and pks5-4 seedlings following a 12-h treatment with $100 \mathrm{mM} \mathrm{NaCl}$. Salt-stressed roots exhibited a pronounced net $\mathrm{Na}^{+}$efflux, however, efflux was significantly lower in pks5-3 and pks5-4 than in BigM (Fig. 3c, d).

To further dissect the function of PKS5-mediated SOS2 phosphorylation, we expressed Myc-SOS2 in pks5-4 (S2) and Myc-SOS2 ${ }^{\mathrm{S} 294 \mathrm{~A}}$ in pks5-4 (S2 ${ }^{\mathrm{SA}}$ ) plants by crossing transgenic Arabidopsis plants expressing 35SPro:6 $\times$ Myc-SOS2 or 35SPro: $6 \times-$ $M y c-S O S 2^{S 294 A}$ with pks5-4. We found that the SOS2 and SOS2 ${ }^{\text {S294A }}$ protein levels were similar in the resulting crossed lines (Supplementary Fig. 3e). Overexpression of SOS2 did not rescue the pks5-4 salt-sensitive phenotype; however, the salt-sensitive phenotype of $p k s 5-4$ was rescued by the expression of SOS2 $2^{\mathrm{S} 294 \mathrm{~A}}$ (Fig. 3e, f), with different independent transgenic lines showing a similar phenotype (Supplementary Fig. 3c, d). We next measured $\mathrm{Na}^{+}$flux in the crossed lines, and found that SOS2 $2294 \mathrm{~A}$ expression rescued the faulty $\mathrm{Na}^{+}$flux of $p k s 5-4$, whereas expression of SOS2 did not (Fig. $3 \mathrm{~g}, \mathrm{~h}$ ).

Thus, PKS5 negatively regulates salt tolerance in Arabidopsis in a manner dependent on SOS2 ${ }^{\text {Ser294 }}$ phosphorylation.

Salt stress enhances the interaction between 14-3-3 and PKS5. SOS2 is activated by salt stress ${ }^{17}$. As we found that PKS5 represses SOS2 activity and enhances the 14-3-3-SOS2 interaction, and that PKS5-mediated suppression of SOS2 in yeast is 143-3 dependent, we hypothesized that PKS5 represses SOS2 activity under normal growth conditions and that salt stress relieves this repression. Thus, we examined whether salt stress affects the kinase activity of PKS5 in planta by generating 
a

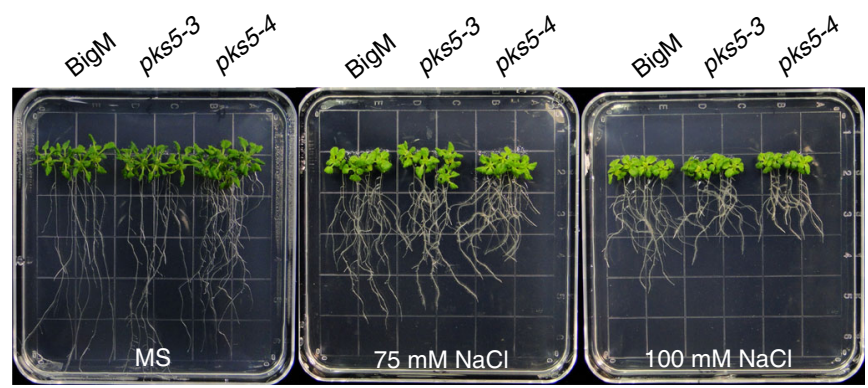

b

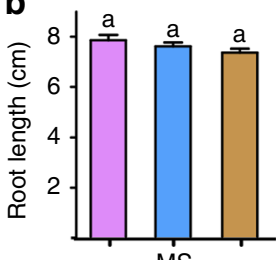

MS

e

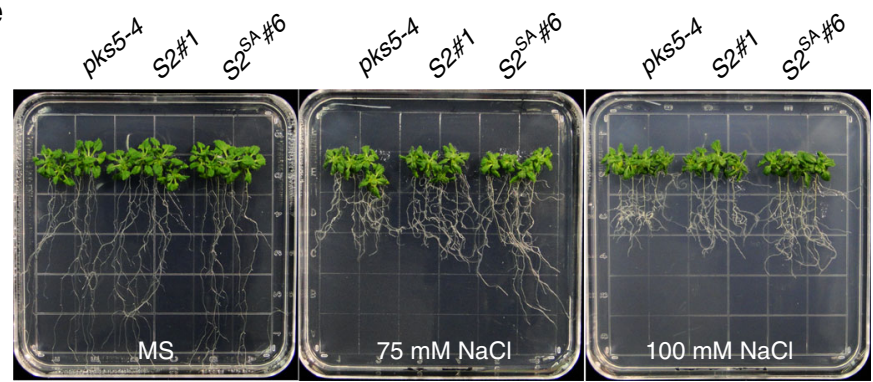

f

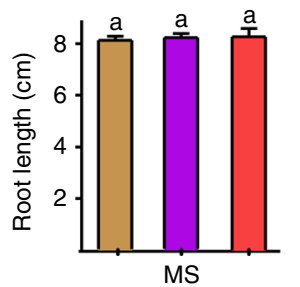

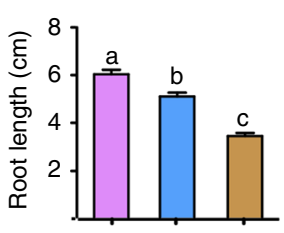

$75 \mathrm{mM} \mathrm{NaCl}$

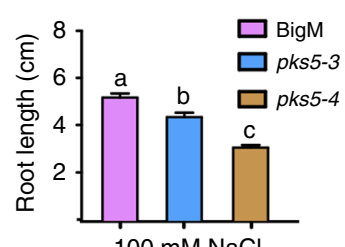

$100 \mathrm{mM} \mathrm{NaCl}$

$100 \mathrm{mM} \mathrm{NaCl}$
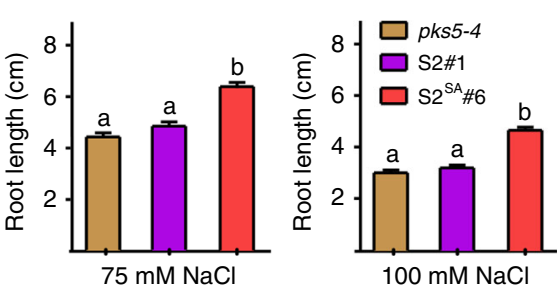

C

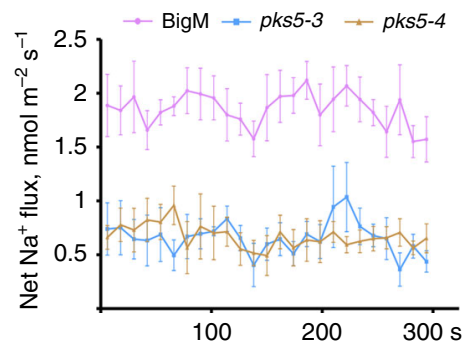

d

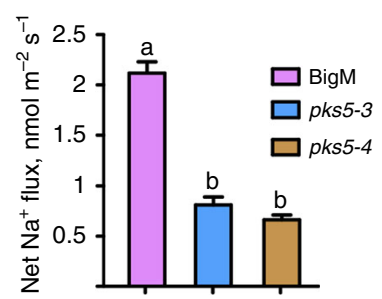

g

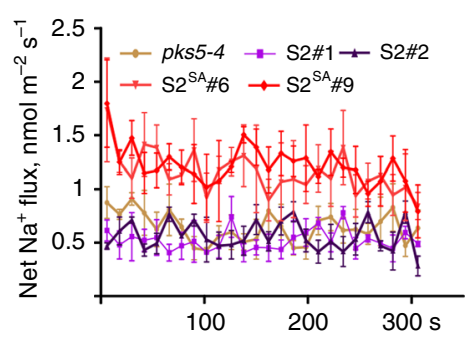

h

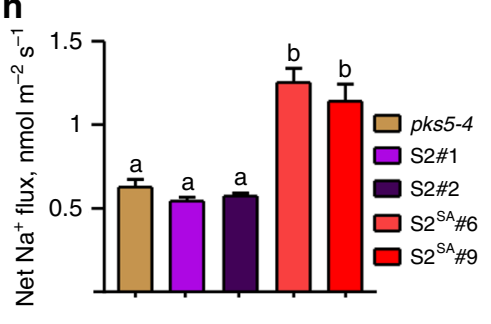

Fig. 3 SOS2 $2^{\text {Ser } 294}$ mutation rescues the salt-sensitive phenotype of pks5-4. a Salt sensitive analysis of BigM, pks5-3, and pks5-4. Five-day-old seedlings were transferred from MS to medium containing 75 or $100 \mathrm{mM} \mathrm{NaCl}$. Photographs were taken 10 days after transfer. b Root length analysis of seedlings in (a). Error bars represent SD; $p \leq 0.05$, Student's $t$-test; $n=12$. c Net Na+flux analysis at the meristem zone of BigM, pks5-3, and pks5-4 by NMT. Five-day-old seedlings were pretreated with $100 \mathrm{mM} \mathrm{NaCl}$ for $24 \mathrm{~h}$. Error bars represent SD; $p \leq 0.05$, Student's $t$-test; $n=6$. d Calculated net Na+ fluxes from (c). e Salt sensitive analysis of pks5-4 and transgenic plants expressing Myc-SOS2 or Myc-SOS2S294A in the pks5-4 background. S2, Pro35S:6×Myc-SOS2 in pks5-4; S2 SA, Pro35S:6×Myc-SOS2S294A in pks5-4. Photographs were taken 10 days after transfer. $\mathbf{f}$ Root length analysis of seedlings in (e). Error bars represent SD; $p \leq 0.05$, Student's $t$-test; $n=12$. $\mathbf{g}$ Net Na+ flux analysis at the meristem zone of $p k s 5-4$ and different transgenic plants by NMT. Error bars represent SD; $p \leq 0.05$, Student's $t$-test; $n=6$. $\mathbf{h}$ Calculated net $\mathrm{Na}^{+}$fluxes from $(\mathbf{g})$. For $\mathbf{b}, \mathbf{d}, \mathbf{f}$, and $\mathbf{h}$, significant differences are indicated by different lowercase letters. Source data of $\mathbf{b}-\mathbf{d}$ and $\mathbf{f}-\mathbf{h}$ are provided in the Source Data file

transgenic Arabidopsis plants overexpressing Myc-PKS5 in Col-0, with expression driven by the $35 \mathrm{~S}$ promoter. Using anti-C-Myc agarose, Myc-PKS5 protein was immunoprecipitated from plants treated with $100 \mathrm{mM} \mathrm{NaCl}$ for various periods and subjected to kinase assay, which showed that $\mathrm{NaCl}$ treatment repressed the kinase activity of PKS5 (Fig. 4a).

We then sought to identify which protein(s) repress PKS5 kinase activity under salt stress. Salt stress reduces the interaction between SOS2 and 14-3-3 proteins ${ }^{21}$, and PKS5 and SOS2 belong to the same sucrose non-fermenting-1-related protein kinase-3 (SnRK3) protein family. We thus hypothesized that 14-3-3 proteins repress the kinase activity of PKS5 under salt stress.
To test this hypothesis, we analyzed the interaction between 14-3-3 $\lambda$ and PKS5 in a yeast two-hybrid assay. We found that 14-3$3 \lambda$ interacted with the N-terminal kinase domain of PKS5 (PKS5N, amino acids 1-280) but not the junction domain (PKS5JD, amino acids 281-306) or C-terminal regulatory domain (PKS5C, amino acids 307-435) (Fig. 4b). Co-immunoprecipitation assays confirmed that PKS5 interacted with 14-3-3 $\lambda$, and that this interaction was induced by $\mathrm{NaCl}$ treatment (Fig. 4c).

A LUC imaging assay confirmed that PKS5 interacted with 143-3 $\lambda$ and that $\mathrm{NaCl}$ treatment enhanced this interaction (Fig. $4 \mathrm{~d}$, e), thus further supporting our conclusion that salt stress enhances the interaction between 14-3-3 proteins and PKS5. 
a

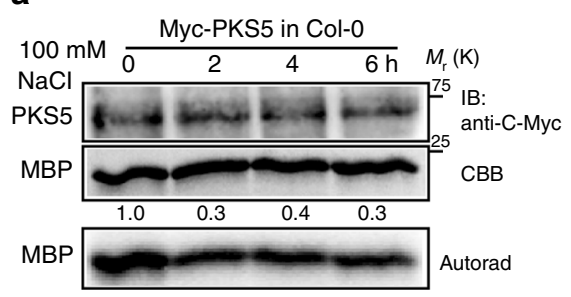

b

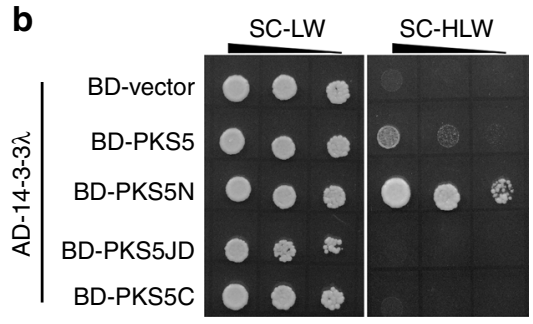

C

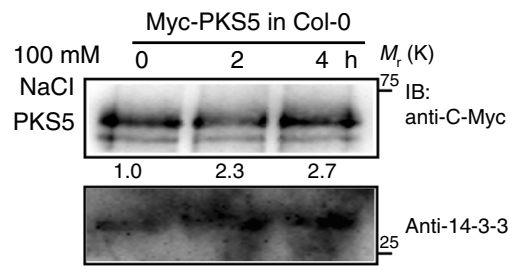

d

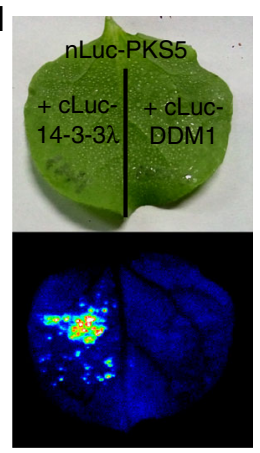

e

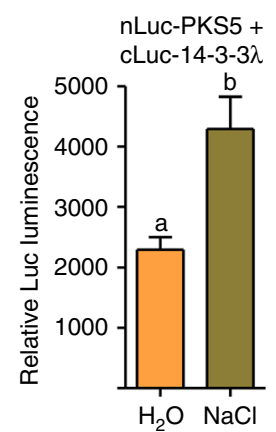

Fig. 4 Salt stress enhances the interactions between 14-3-3 proteins and PKS5. a In vitro kinase assay shows that salt stress decreases PKS5 activity. MycPKS5 was extracted from 10-day-old Col-0 seedlings transgenically expressing Pro35S:6×Myc-PKS5 treated with $100 \mathrm{mM} \mathrm{NaCl}$ for the indicated periods.

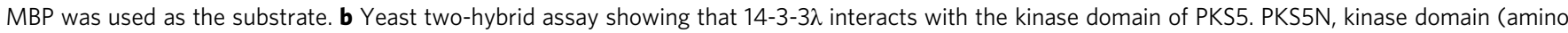
acids 1-280); PKS5JD, junction domain (amino acids 281-306); PKS5C, C-terminal regulatory domain (amino acids 307-435). c In vivo Co-

immunoprecipitation assay showing that salt stress enhances the interaction between PKS5 and 14-3-3 proteins. Myc-PKS5 was extracted from Col-0 expressing Pro35S:6×Myc-PKS5 treated with $100 \mathrm{mM} \mathrm{NaCl}$ for the indicated periods, and then analyzed by immunoblotting with anti-C-Myc and anti-14-3-3 antibodies. d PKS5 interacts with 14-3-3 $\lambda$ in a Luciferase complementation assay. DDM1 was used as a negative control. e Luciferase complementation

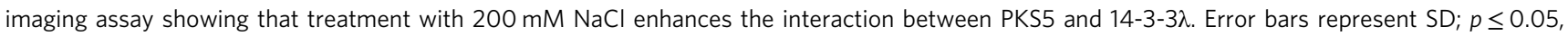
Student's $t$-test; $n=6$; significant difference is indicated by different lowercase letters. CBB Coomassie Brilliant Blue, Autorad autoradiograph, IB immunoblot. Source data of $\mathbf{a}, \mathbf{c}$, and $\mathbf{e}$ are provided in the Source Data file

14-3-3 proteins repress the kinase activity of PKS5. The finding that salt stress reduces the kinase activity of PKS5 and increases the interaction between 14-3-3 $\lambda$ and PKS5 led us to hypothesize that $14-3-3 \lambda$ may directly repress PKS5 activity via protein-protein interaction under salt stress. To test this hypothesis, we performed an in vitro kinase assay using recom-

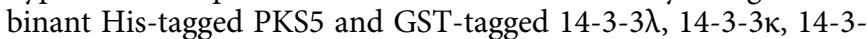
$3 \omega$, and SCaBP1 (a substrate for PKS5) from Arabidopsis following heterologous expression and purification from $E$. coli. We

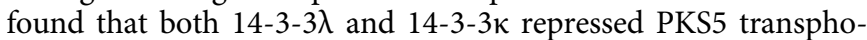
sphorylation activity against SCaBP1 and also repressed PKS5 autophosphorylation. 14-3-3 $\omega$, included as a control, did not markedly affect SCaBP1 phosphorylation (Fig. 5a) and the inhibition of SCaBP1 phosphorylation by $14-3-3 \lambda$ was dose dependent. To further investigate the effect of 14-3-3 proteins on PKS5 activity in vivo, we performed an IP-kinase assay. We generated transgenic Arabidopsis plants overexpressing $M y c-P K S 5$ in the

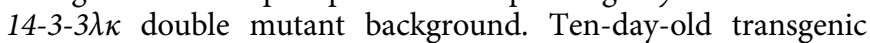
seedlings were treated with $100 \mathrm{mM} \mathrm{NaCl}$ for 2 or $4 \mathrm{~h}$, and then PKS5 was immunoprecipitated by anti-C-Myc agarose. Consistent with previous results (Fig. 4a), the phosphorylation of MBP was repressed in Col-0 by salt stress (Fig. 5b); however, no significant difference in PKS5 kinase activity was observed after $\mathrm{NaCl}$ treatment between the $14-3-3 \lambda \kappa$ double mutant and Col-0 (Fig. 5c). These results suggest that 14-3-3 proteins repress PKS5 activity under salt stress.

Previous studies suggest that PKS5 negatively regulates $\mathrm{PM} \mathrm{H} \mathrm{H}^{+}$-ATPase activity, and that the PKS5 loss-of-function mutant $p k s 5-1$ is tolerant to high external $\mathrm{pH}^{24}$. As $14-3-3 \lambda$ and $\kappa$ both repress PKS5 activity, we examined the growth of $14-3-3 \lambda \kappa$ double mutant seedlings in response to high external $\mathrm{pH}$. On $\mathrm{pH}$ 6.5 and 6.7 MS medium, root elongation was inhibited to a greater extent in the $14-3-3 \lambda \kappa$ double mutant than in Col-0 (Supplementary Fig. 4a, b).

Furthermore, we measured $\mathrm{PM} \mathrm{H} \mathrm{H}^{+}$-ATPase activity using plasma membrane-enriched vesicles ${ }^{29}$, which were isolated from

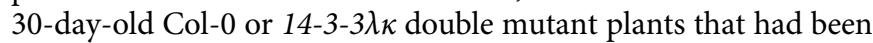
subjected to $\mathrm{NaCl}$ treatment for 3 days. We found that the $\mathrm{NaCl}-$ induced increase in $\mathrm{PM} \mathrm{H}{ }^{+}$-ATPase activity was significantly

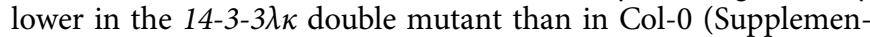
tary Fig. 4c, d). NMT measurement also indicated that the $\mathrm{H}^{+}$ efflux of $14-3-3 \lambda \kappa$ double mutants was lower than that of Col-0 (Supplementary Fig. 4e, f).

Phosphorylation of AHA2 C-terminal $\mathrm{Thr}^{947}$ generates a binding site that promotes interaction with the $14-3-3 \omega$ protein and activation of the $\mathrm{PM} \mathrm{H}^{+}$-ATPase ${ }^{30}$. We examined if 14-3-3 $\lambda$ and $14-3-3 \kappa$ also interact with AHA2. Yeast two-hybrid analysis showed that when $\mathrm{Thr}^{947}$ was mutated to Asp, 14-3-3w interacted with AHA2 $\mathrm{C} 100^{\mathrm{T} 947 \mathrm{D}}$, whereas no interaction was detected

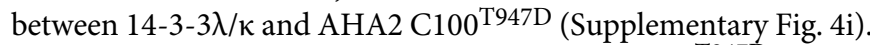
LUC imaging assays also showed that AHA2 C100 T947D (947D) strongly interacted with $14-3-3 \omega$ but not $14-3-3 \lambda, 14-3-3 \kappa$, or the negative control DDM1 in transgenic $N$. benthamiana plants (Supplementary Fig. 4g, h).

In addition, we measured the effect of GST-14-3-3 proteins on $\mathrm{PM} \mathrm{H}^{+}$-ATPase activity using plasma membrane vesicles isolated from Col-0 after 3 days of $\mathrm{NaCl}$ treatment. Consistent with previous results, $14-3-3 \omega$ protein significantly increased $\mathrm{PM} \mathrm{H}^{+}-\mathrm{ATPase}$ activity, whereas $14-3-3 \lambda$ and $14-3-3 \kappa$ proteins had no effect on PM $\mathrm{H}^{+}$-ATPase activity, similar to the 


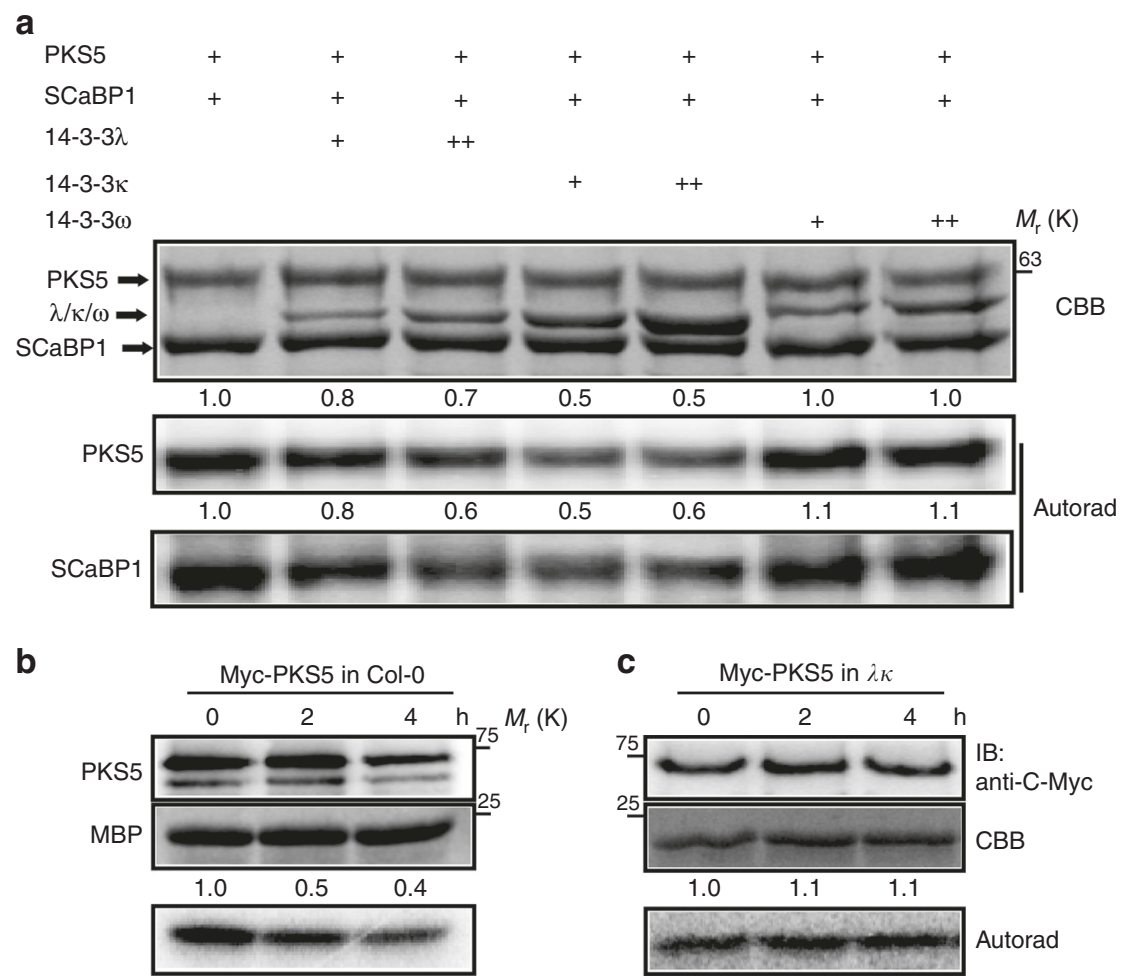

Fig. 5 14-3-3 proteins repress the kinase activity of PKS5. a In vitro kinase assay showing that 14-3-3 $\lambda$ and $\kappa$ proteins inhibit PKS5 activity in a dosedependent manner. Recombinant His-tagged PKS5 was incubated with GST-tagged SCaBP1 with or without GST-tagged 14-3-3 proteins. SCaBP1 was used

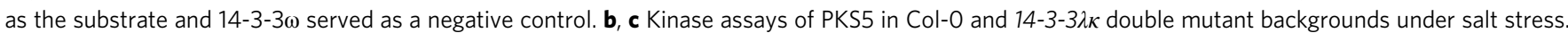
Myc-PKS5 was purified from Pro35s:6×Myc-PKS5 transgenic plants treated with $100 \mathrm{mM} \mathrm{NaCl}$ for the indicated periods. MBP was used as the substrate. CBB Coomassie Brilliant Blue, Autorad autoradiograph, IB immunoblot. Source data are provided as a Source Data file

GST-tagged protein, which was used as a negative control (Supplementary Fig. 4j). These results suggest that $14-3-3 \lambda$ and 14-3-3K regulate PM $\mathrm{H}^{+}$-ATPase activity by repressing PKS5 activity.

14-3-3s function in PKS5-mediated alkaline stress response. Given that PKS5 negatively regulates PM $\mathrm{H}^{+}$-ATPase activity, the loss-of-function pks5-1 mutant showed an alkaline tolerance phenotype, whereas the gain-of-function $p k s 5-3$ and $p k s-4$ mutants showed an alkaline sensitive phenotype ${ }^{29}$. To test whether $14-3-3 \lambda$ and $\kappa$ are required for plant responses to alkaline stress, we crossed Col-0 expressing Pro35S:Flag-HA-14-3-3 $3 \lambda^{21}$ with $p k s 5-1$ or $p k s 5-4$ mutant plants to overexpress $14-3-3 \lambda$ in the pks5-1 (OE-1 and OE-2) and pks5-4 (OE-3 and OE-4) backgrounds. Two independent lines were subsequently used in an

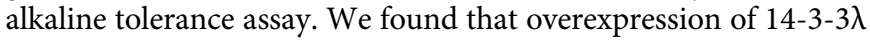
in $p k s 5-4$ repressed the alkaline-sensitive phenotype of the mutant as well as $\mathrm{PM} \mathrm{H}^{+}$-ATPase activity (Fig. 6a-c); however, the alkaline tolerance phenotype and high $\mathrm{PM} \mathrm{H}^{+}$-ATPase activity of pks5-1 were not repressed (Fig. 6d-f). Furthermore, over-

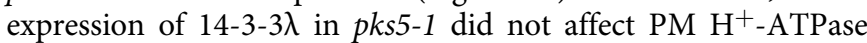

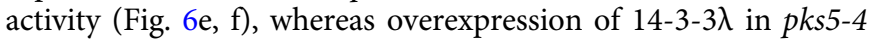
partially rescued $\mathrm{PM} \mathrm{H} \mathrm{H}^{+}$-ATPase activity (Fig. 6b, c). These results further indicate that $14-3-3 \lambda$ and $\kappa$ regulate the alkaline stress response in Arabidopsis at least partly by repressing of PKS5 kinase activity.

14-3-3 binds to calcium that regulates SOS2/PKS5 activity. A salt stress-induced increase in cytosolic $\mathrm{Ca}^{2+}$ concentration decreases the interaction between14-3-3 and SOS2 and enhances the interaction between 14-3-3 and PKS5. It has been reported that $14-3-3 \lambda$ and $\kappa$ contain a conserved $\mathrm{Ca}^{2+}$-binding EF-hand motif $^{31}$. Thus, we hypothesized that $14-3-3 \lambda$ and $\kappa$ play a role in regulating PKS5 and SOS2 kinase activity through calcium signals. We first tested whether 14-3-3 proteins can bind $\mathrm{Ca}^{2+}$ in a microscale thermophoresis (MST) assay, and found that, in contrast to the negative control (GST-tagged protein), GST-14-3$3 \lambda$ and GST-14-3-3k bound $\mathrm{Ca}^{2+}$ directly, the calcium-binding protein CDPK6 served as a positive control (Fig. 7a). In vitro kinase assays suggest $\mathrm{Ca}^{2+}$ released SOS2 kinase activity from 143-3-mediated inhibition (Fig. 7b; Supplementary Fig. 5e). By contrast, the addition of $\mathrm{Ca}^{2+}$ repressed PKS5 kinase activity (Fig. 7c; Supplementary Fig. 5f). The addition of the same amount of $\mathrm{Ca}^{2+}$ did not directly affect the activities of PKS5 and SOS2 (Supplementary Fig. 5g, h). Furthermore, we found that the saltenhanced 14-3-3/PKS5 and SOS3/SOS2 interactions were rescued by the addition of $\mathrm{LaCl}_{3}$, a calcium channel blocker (Supplementary Fig. 5a-d) and that the effects of $\mathrm{Ca}^{2+}$ on the repression of PKS5 and activation of SOS2 activities were rescued by EGTA (Supplementary Fig. 5e, f). These results suggest that the saltinduced calcium signal may positively regulate the 14-3-3/PKS5 interaction and regulates the activity of PKS5 and SOS2 kinases.

Previous studies demonstrated that 14-3-3 $\omega$ binds to calcium at its C-terminus via an EF-hand-like motif ${ }^{32}$. We analyzed eight 14-3-3 homologs in Arabidopsis that contain a putative EF-hand, and found that $14-3-3 \lambda^{\mathrm{G}} 216$ is the only conserved amino acid in 8 of 13 14-3-3 proteins (Supplementary Fig. 6a). We mutated this residue to Ala and purified the recombinant protein from E. coli, and following a test of $\mathrm{Ca}^{2+}$-binding activity by MST assays, found that 14-3-3 $\lambda^{\mathrm{G} 216 \mathrm{~A}}$ did not bind to $\mathrm{Ca}^{2+}$ (Fig. 7d).

Yeast two-hybrid and LUC imaging assays showed that the 143-3 $3 \lambda^{\mathrm{G} 216 \mathrm{~A}}$ mutation had no obvious effect on its ability to interact with PKS5 and SOS2, with protein interaction levels observed for 14-3-3 $\lambda^{\mathrm{G} 216 \mathrm{~A}}$ being comparable to those of wild-type $14-3-3 \lambda$ 


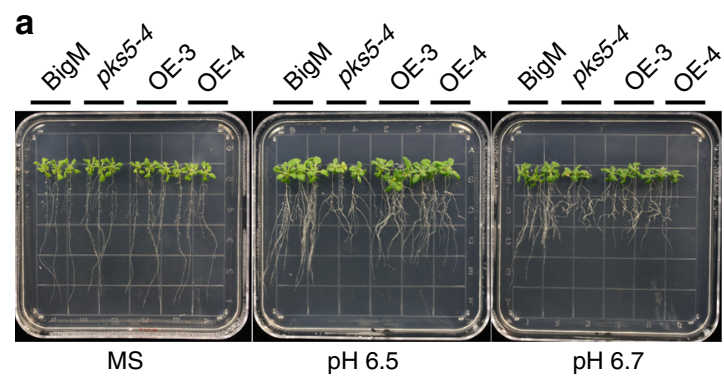

d

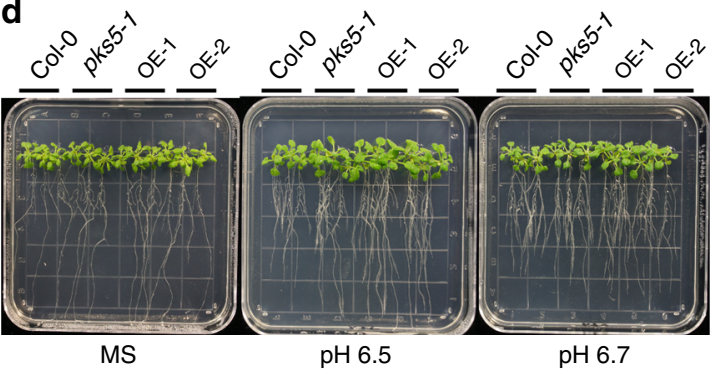

b

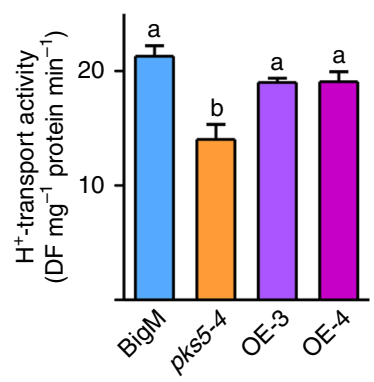

e

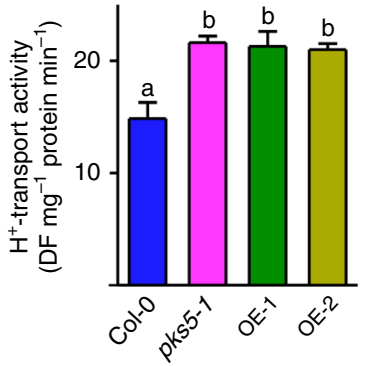

C

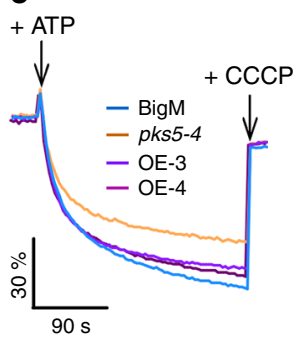

f

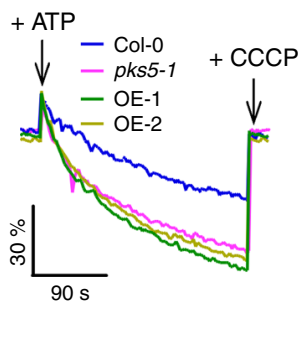

Fig. 6 Overexpression of 14-3-3 rescues the phenotypes of pks5-4. a Alkaline sensitive analysis of BigM, pks5-4, and two independent transgenic lines (OE-

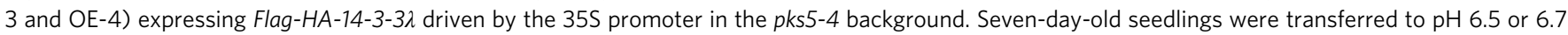
MS medium adjusted using $\mathrm{NaHCO}_{3}$. Photographs were taken 10 days after seedling transfer. b Comparison of PM H+-ATPase activity in BigM, pks5-4, $\mathrm{OE}-3$, and OE-4. Plasma membrane vesicles were purified from 5-week-old seedlings grown in soil treated with $250 \mathrm{mM} \mathrm{NaCl}$ for 3 days. Error bars represent SD; $p \leq 0.05$, Student's $t$-test; $n=3$; significant differences are indicated by different lowercase letters. c Timely varying curves of $\mathrm{PM} \mathrm{H}^{+}$-ATPase activity in BigM, pks5-4, OE-3, and OE-4. d Alkaline tolerance analysis of Col-0, pks5-1, and two independent transgenic lines (OE-1 and

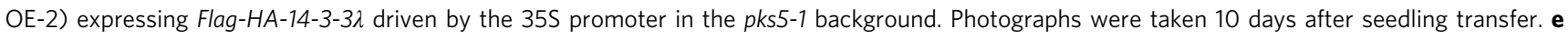
Comparison of PM H+-ATPase activity in Col-0, pks5-1, OE-1, and OE-2. Error bars represent SD; $p \leq 0.05$, Student's $t$-test; $n=3$; significant differences are

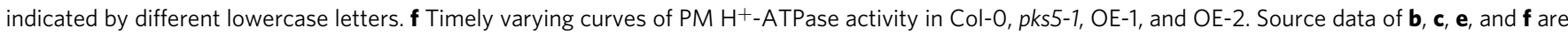
provided in the Source Data file

(Supplementary Fig. 6b, d). And transient gene expression levels of $14-3-3 \lambda, 14-3-3 \lambda^{\mathrm{G} 216 \mathrm{~A}}$, PKS5, and SOS2 in the LUC-imaging assay were similar (Supplementary Fig. 6c). Furthermore, we tested if calcium affects the ability of $14-3-3 \lambda$ G216A to repress SOS2 and PKS5 kinase activity. In vitro kinase assays using 14-3$3 \lambda^{\mathrm{G} 216 \mathrm{~A}}$ showed that the addition of $\mathrm{Ca}^{2+}$ did not change the 14$3-3 \lambda$ G216A-mediated repression of PKS5 and SOS2 activities (Fig. 7e, f), although weak repressive activity towards SOS2 and PKS5 was observed when using 14-3-3 $\lambda^{\mathrm{G} 216 \mathrm{~A}}$ (Supplementary Fig. 6e). Taken together, our results suggest that different calcium concentrations are involved in regulating 14-3-3-mediated SOS2 and PKS5 activation and repression.

\section{Discussion}

The calcium signal-activated SOS pathway is a conserved and important pathway that mediates plant sodium ion homeostasis under salt stress. According to previous studies and the results presented here, we propose a working model of how 14-3-3 proteins decode a calcium signal to enhance the salt tolerance of plants (Fig. 7g). Under normal growth conditions, PKS5 may phosphorylate SOS2 $2^{\mathrm{Ser} 294}$, and promote the 14-3-3/SOS2 interaction that in turn could inhibit SOS2 kinase activity and limit SOS1 $\mathrm{Na}^{+} / \mathrm{H}^{+}$antiporter activity to basal levels. Furthermore, PKS5 can phosphorylate $\mathrm{PM} \mathrm{H}^{+}$-ATPases, thus limiting their activity, which is required to provide the driving force for the $\mathrm{Na}^{+} / \mathrm{H}^{+}$antiporter activity of SOS1. It is important that, under 'none-stressed' growth conditions, the proton ATPase maintains membrane potential and solute/nutrient uptake.
Therefore, precise regulation of $\mathrm{PM} \mathrm{H}^{+}$-ATPase activity is important for plant growth and stress response. Under salt stress, the salt-induced calcium signal is decoded by various $\mathrm{Ca}^{2}$ +-binding proteins, including 14-3-3s, SOS3, and SCaBP8, which may result in increased repression of PKS5 activity that reduces SOS2 2 rer294 phosphorylation and in turn, relieves the repression of SOS2 activity by 14-3-3 proteins. In addition, a previous study reported that the $\mathrm{AHG} 3$, a member of $\mathrm{PP} 2 \mathrm{Cs}$, interacts with SnRK3.22 (PKS5) ${ }^{33}$, which may participate in the regulation of PKS5 kinase activity. SOS3 and SCaBP 8 bind to $\mathrm{Ca}^{2+}$ ions, and then recruit SOS2 to the plasma membrane and activate it. SOS2 activity results in the activation of the $\mathrm{PM} \mathrm{Na}+/ \mathrm{H}^{+}$antiporter SOS1, and reduced PKS5 activity triggers the activation of PM H +-ATPase by binding to 14-3-3w, which provides SOS1 with the proton gradient needed to drive $\mathrm{Na}^{+}$transportation (Fig. 7i). However, it is not clear whether and how calcium and calciumbinding protein SCaBPs/CBLs play roles in regulating PKS5 and SOS2 under normal growth conditions, and whether and how they function in deactivation of PKS5 under salt stress.

Previous studies suggest that J3 (DnaJ homolog 3) interacts with and represses PKS5 kinase activity under the normal growth condition, which in turn activates the $\mathrm{PM} \mathrm{H}^{+}$-ATPase activity ${ }^{29}$. $\mathrm{J} 3$ interacts with the regulatory domain of PKS5 (PKS5C), and 143-3 proteins interacted with the kinase domain of PKS5 (PKS5N), which suggests that $14-3-3$ proteins may directly repress PKS5 kinase activity, whereas J3 may change the structure of PKS5 and then represses the kinase activity of PKS5. However, it is not fully understood whether 14-3-3 and J3 repress PKS5 in different times or stress conditions or they work together to repress PKS5. 
a

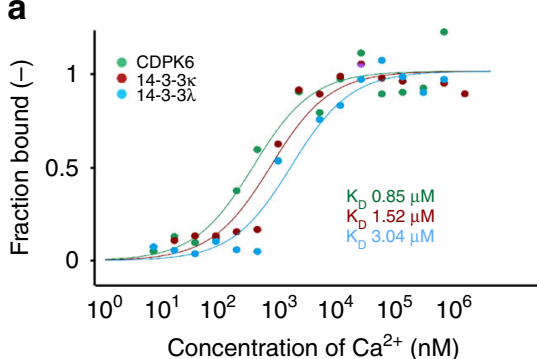

d

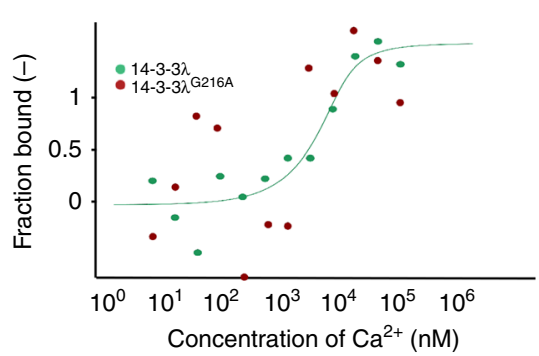

b

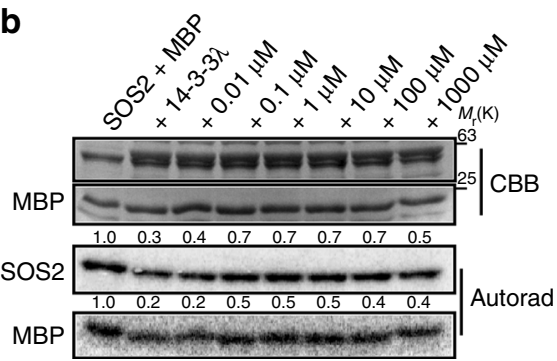

$\mathbf{e}$

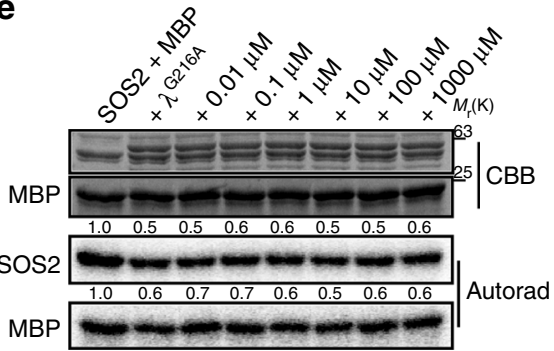

C

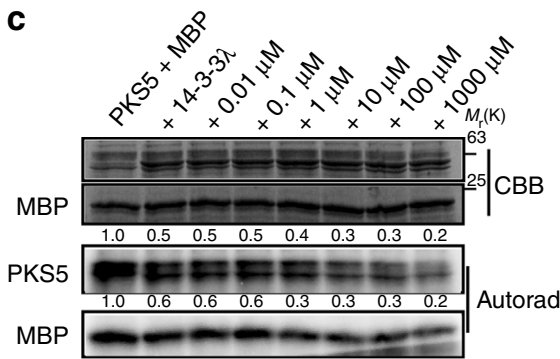

f

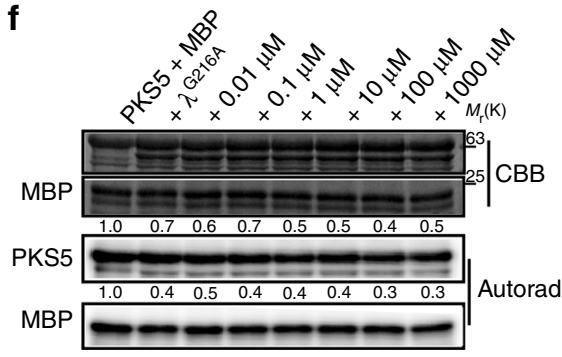

g

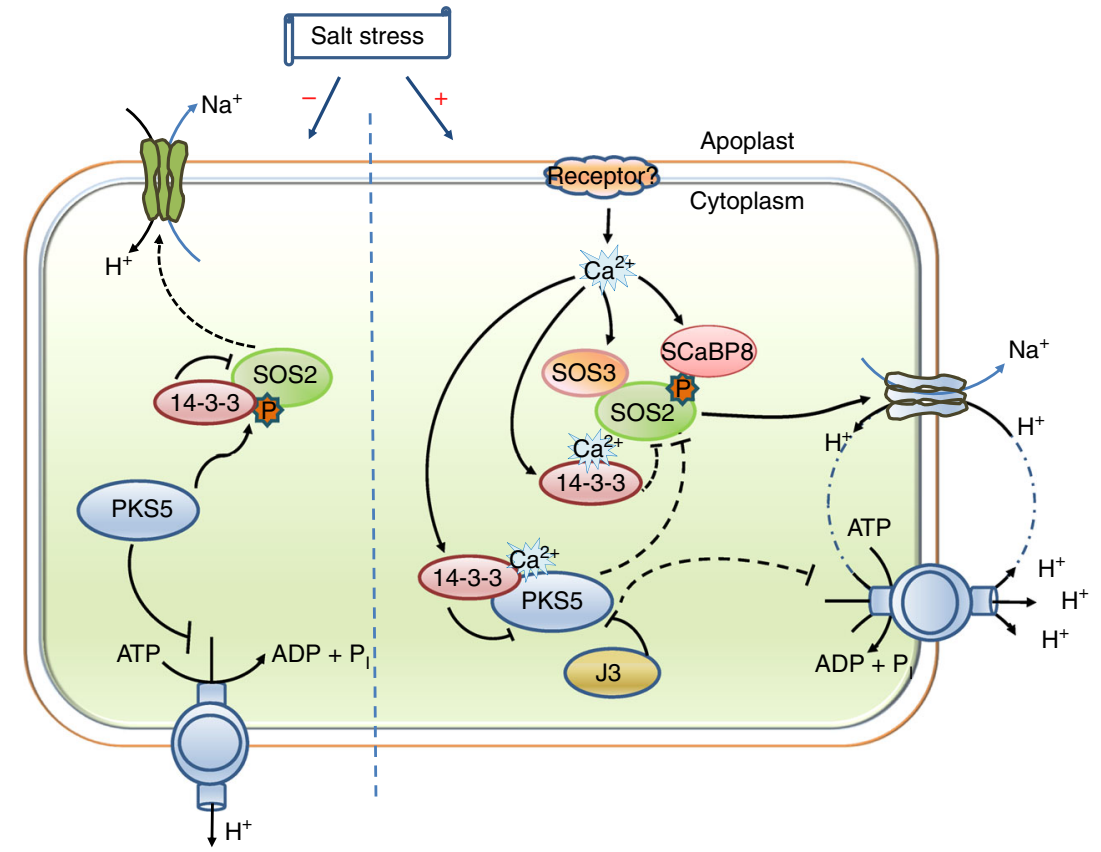

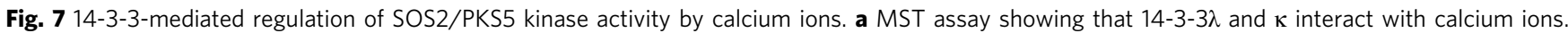
CDPK6 served as a positive control. Data are representative of three independent biological experiments. $\mathbf{b}$ In vitro kinase assay showing that $\mathrm{Ca}^{2+}$ decreases the 14-3-3-mediated repression of SOS2. $\mathbf{c}$ In vitro kinase assay showing that $\mathrm{Ca}^{2+}$ enhances the 14-3-3-mediated repression of PKS5. $\mathbf{d}$ MST assay of the $\mathrm{Ca}^{2+}$-binding affinity of $14-3-3 \lambda^{\mathrm{G} 216 \mathrm{~A}}$. Data are representative of three independent biological experiments. e In vitro kinase assay showing that the $14-3-3 \lambda$ G216A mutant does not significantly repress SOS2 activity in the presence of $\mathrm{Ca}^{2+} . \mathbf{f}$ In vitro kinase assay showing that the $14-3-3 \lambda^{\mathrm{G} 216 \mathrm{~A}}$ mutant does not significantly repress PKS5 activity in the presence of $\mathrm{Ca}^{2+}$. $\mathbf{g}$ Model illustrating how 14-3-3 proteins decode a calcium signal to enhance the salt tolerance of plants. Under normal growth conditions, PKS5 phosphorylates SOS2 ${ }^{S e r 294}$ and represses SOS2 activity. Upon salt stress, 14-3-3 proteins decode a salt-induced calcium signal, repress PKS5, and release SOS2 to activate PM H $\mathrm{H}^{+}$-ATPase and SOS1, respectively. For $\mathbf{b}$ and $\mathbf{c}$, about $1.0 \mu \mathrm{gg}$

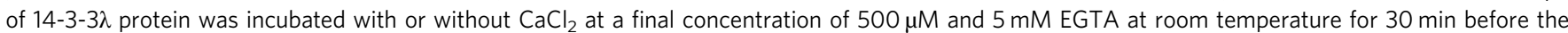
kinase assay. MBP was used as a substrate. CBB Coomassie brilliant blue, Autorad autoradiograph. Source data of a-f are provided in the Source Data file

To date, many studies have shown that 14-3-3 proteins play crucial roles in a variety of biological processes, in plants, and are key regulators of various signal transduction pathways. For instance, they transduce the cold signal into the nucleus and thereby induce the expression of cold responsive genes in Arabidopsis $^{34}$, and transduce the BR signal by localizing BZR1 to the nucleus in Arabidopsis and rice (Oryza sativa) ${ }^{35}$. In this study, we found that 14-3-3 proteins transmit salt-induced calcium signals to SOS2/PKS5 kinases and coordinately relieve/inhibit their activity, which triggers $\mathrm{Na}^{+} / \mathrm{H}^{+}$antiporter and $\mathrm{PM} \mathrm{H}{ }^{+}$-ATPases activity, and thereby maintains sodium ion homeostasis under salt stress. 
In Arabidopsis, 8 out of 13 14-3-3 homologs, including $14-3-3 \lambda, \kappa$, and $\omega$, were reported to contain an EF-hand motif 31 , supporting the notion that calcium signals regulate 14-3-3mediated SOS2 and PKS5 protein kinase inhibition. Activation of TMPK1 kinase by calcium ions requires $14-3-3 \varphi^{36}$ and $14-3-3 \omega$ binds calcium at its highly conserved C-terminus ${ }^{32}$. A mutation in the 14-3-3 $\lambda$ EF-hand motif that abolished its calcium-binding activity did not rescue the 14-3-3 mutant phenotype and removed

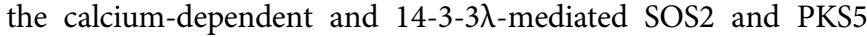
kinase repression, demonstrating that calcium binding is essential

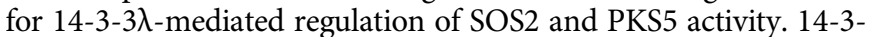
$3 \omega$ activates $\mathrm{PM} \mathrm{H}^{+}$-ATPases by binding to the penultimate $\mathrm{C}$ terminal phosphorylated $\mathrm{Thr}^{947}$, and a calcium signal may also be required for this regulation ${ }^{37,38}$. Phosphorylation of AHA2 Ser ${ }^{931}$ by PKS5 blocks this binding, thereby inhibiting AHA2 activity ${ }^{24}$. However, 14-3-3 $\omega$ does not interact with PKS5. These results indicate that the salt-induced calcium signal also coordinately regulates different 14-3-3 proteins to activate $\mathrm{PM} \mathrm{H}^{+}$-ATPase.

$14-3-3 \lambda$ and $\kappa$ bind to the junction domain of SOS2 and phosphorylation of Ser ${ }^{294}$ enhances this binding ${ }^{21}$; however, 14-33 proteins interact with the kinase domain of PKS5, which contains no conserved 14-3-3-binding motif, suggesting that 14-3-3 proteins play different roles in regulating SOS2 and PKS5 activity, and that the underlying regulatory mechanism may differ.

Interestingly, the 14-3-3 $\lambda \kappa$ double mutant displayed high levels of $\mathrm{Na}^{+}$efflux (Supplementary Fig. 6f, g) and low levels of $\mathrm{H}^{+}$ efflux (Supplementary Fig. 4e, f). Consistent with this observation, the mutant is more tolerant to sodium ${ }^{21}$ and more sensitive to alkaline conditions than the wild type. However, activation of PM $\mathrm{H}^{+}$-ATPase is required for enhancing $\mathrm{Na}^{+} / \mathrm{H}^{+}$antiporter activity. We compared the salt sensitivity of $14-3$-3 $\lambda$-overexpression lines

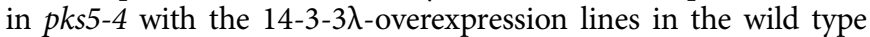
(BigM), and found that the overexpression of $14-3-3 \lambda$ in BigM resulted in the transgenic plants were more sensitive to salt treatment compared with that of BigM (Supplementary Fig. 7a, b). However, overexpressing 14-3-3 $\lambda$ in pks5-4 could not rescue the salt-sensitive phenotype of pks5-4 (Supplementary Fig. 7a, b), although the $14-3-3 \lambda$ protein levels were similar in these overexpression lines (Supplementary Fig. 7c).These results suggest that a certain degree of $\mathrm{PM} \mathrm{H}^{+}$-ATPase activity is enough to provide the driving force for $\mathrm{PM} \mathrm{Na} \mathrm{Na}^{+}$antiporter activity and that SOS2 is more important for this activation.

Taken together, these findings suggest that switch-like 14-3-3 proteins bind to and repress SOS2 or PKS5 in the absence or presence of salt and that these protein-protein interactions are associated with an increased 14-3-3 calcium-binding affinity, which is critical for the plant's ability to adapt to salt stress.

\section{Methods}

Plant materials and growth conditions. Arabidopsis Col- 0 and Col erecta105 (BigM) plants were used in this study, $p k s 5-3$ and $p k s 5-4$ are in BigM background, and the others are in Col-0 background. The reported materials pks5-1, pks5-3,

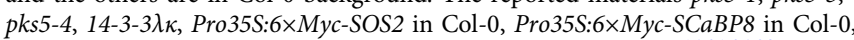

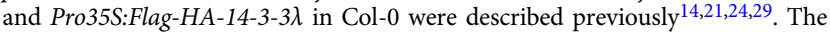
Pro35S:6×Myc-SOS2/SCaBP8 in BigM/pks5-1/pks5-3/pks5-4 and Pro35S:6 $\times M y c-$

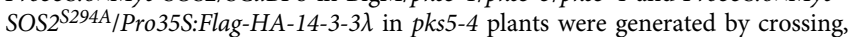
and two homozygous transgenic lines were detected by anti-C-Myc (CWBIO 01217/10153, 1/3000), anti-HA (Sigma-Aldrich H3663, 1/3000), or anti-Actin (CWBIO 01265/60205, 1/5000), and used for further studies. All the plants were grown at $22-24^{\circ} \mathrm{C}$ under a 16 -light and 8 -h dark photoperiod. Seeds were imbibed at $4{ }^{\circ} \mathrm{C}$ for 2 days and geminated on Murashige-Skoog (MS) Petri dishes containing $0.5 \%$ phytagel and $2.0 \%$ sucrose in a light growth chamber.

Mass spectrometry analysis. The 10-day-old Pro35S:Flag-HA-SOS2 seedlings (about $5 \mathrm{~g}$ ) in the sos2-2-mutant background ${ }^{21}$ grown on MS Petri dishes were collocated and ground to a fine powder in liquid nitrogen. The plant material was incubated in IP buffer for $30 \mathrm{~min}$ on ice $(10 \mathrm{mM}$ Tris, $\mathrm{pH} 7.5,0.5 \%$ Nonidet P-40, $2 \mathrm{mM}$ EDTA, $150 \mathrm{mM} \mathrm{NaCl}, 1 \mathrm{mM}$ PMSF, and $1 \%$ protease inhibitor cocktail, Roche) and centrifuged at $12,000 \times g$ at $4{ }^{\circ} \mathrm{C}$ for $10 \mathrm{~min}$ to remove cell debris. The
anti-HA-conjugated agarose (Sigma-Aldrich) was used for HA-SOS2 immunoprecipitation. The SOS2 protein and its interacting proteins were then analyzed by a LTQ ORBITRAP Velos mass spectrometer (ThermoFisher Scientific). Database searches were performed on an in-house Mascot server (Matrix Science Ltd.) against International Protein Index (IPI) Arabidopsis database. The matched peptides were filtered with 0.05 significance threshold. The Mascot Percolator scores were used for all peptides.

To confirm the phosphorylation site of SOS2 ${ }^{\mathrm{Ser} 294}$ site by PKS5, samples containing $15 \mu \mathrm{g}$ of His-tagged SOS2 and His-tagged PKS5 retained on beads. Total proteins were incubated in $30 \mu \mathrm{L}$ of kinase buffer $(20 \mathrm{mM}$ Tris- $\mathrm{HCl}, \mathrm{pH} 8.0,1 \mathrm{mM}$ DTT, $5 \mathrm{mM} \mathrm{MgCl}_{2}$, and $10 \mu \mathrm{M} \mathrm{ATP}$ ) at $30^{\circ} \mathrm{C}$ for $30 \mathrm{~min}$, His-tagged PKS5 were removed by a 5 -min centrifugation at $500 \times g$ at $4{ }^{\circ} \mathrm{C}$. His-tagged SOS 2 were collected and analyzed by LC-MS/MS.

Plasmids construction and recombinant proteins. To generate recombinant protein expression plasmid constructs, the coding sequences of SOS2-JD ${ }^{\text {S294A, }}$,

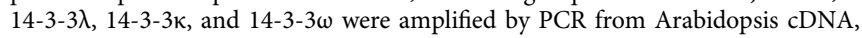
and the resulting cDNA fragments were cloned into the BamHI and SalI sites of $p G E X-6 p-1$ vector. The GST fusion recombinant proteins were expressed in E. coli DE3 strains. The bacteria were grown in liquid LB medium at $37^{\circ} \mathrm{C}$ to an $\mathrm{OD}_{600}$ of $1.0-1.5$. The recombinant proteins were induced by $0.2 \mathrm{mM}$ isopropyl $\beta-\mathrm{D}-1-$ thiogalactopyranoside (IPTG) and purified with glutathione sepharose (MCLAB) His-tagged SOS2 and PKS5 were purified by nickel-nitrilotriacetic acid agarose (MCLAB).

Yeast two-hybrid assays. To analyze the interaction between PKS5 and SOS2 or 14-3-3 $\lambda$, the coding sequences of PKS5, PKS5N, PKS5JD, and PKS5C were cloned into the $p G B K T 7$ vector, and the resulting vectors were co-transformed with

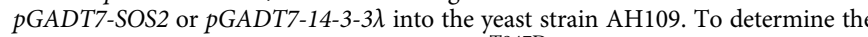
interaction between $14-3-3 \mathrm{~s}$ and AHA2 $\mathrm{C} 100^{\mathrm{T} 947 \mathrm{D}}$, the coding sequences of 14-3$3 \kappa$ and $14-3-3 \omega$ were cloned into $p G A K T 7$ vector, the AHA2 C100 ${ }^{\text {9947D }}$ coding sequence was cloned into $p G B K T 7$ vector, and the resulting vectors were transformed into AH109. SC/-Trp/-Leu or SC/-Trp/-Leu/-His medium were used to detect the interactions.

In vitro kinase assays. An in vitro kinase assay was performed as described below. The recombinant proteins were purified from E. coli DE3 strains and the Myctagged proteins were immunoprecipitated from transgenic plants. For the kinase reaction, $25 \mu \mathrm{L}$ of reaction mixtures with $1 \mu \mathrm{Ci}$ of $\left[\gamma^{-32} \mathrm{P}\right]$ ATP in kinase buffer (20 mM Tris- $\mathrm{HCl}, \mathrm{pH} 8.0,1 \mathrm{mM}$ DTT, $5 \mathrm{mM} \mathrm{MgCl}_{2}$, and $10 \mu \mathrm{M} \mathrm{ATP}$ ) were prepared. The mixtures were kept at $30^{\circ} \mathrm{C}$ for $30 \mathrm{~min}$ and $6 \times$ SDS loading buffer was used to stop the reactions. Proteins were separated by a $10 \%$ or $12 \%$ SDS-PAGE gel, the phosphorylation signals were visualized by a Typhoon 9410 imager and quantified by ImageJ software.

Bimolecular fluorescence complementation. BiFC assays were performed using $N$. benthamiana as described below. The coding sequences of PKS5 and SOS2 were cloned into $p S P Y N E(R) 173$ and $p S P Y C E(M)$ vectors, respectively. The resulting constructs were transformed into Agrobacterium strain GV3101 and then infiltrated into $N$. benthamiana leaves. Three days later, the YFP fluorescence was detected by Leica confocal.

LUC assay. The coding sequences of PKS5 and AHA2 C100 $947 \mathrm{D}$ were cloned into the $p C A M B I A-n L u c$ vectors, the coding sequences of $14-3-3 \kappa$ and $14-3-3 \omega$ were cloned into the $p C A M B I A-c L u c$ vectors, and the nLuc-SOS2 and cLuc-14-3-3 $\lambda$ has been described ${ }^{21}$. All these constructs were introduced into Agrobacterium strain GV3101, and then infiltrated into N. benthamiana leaves as described below. Two days after infiltration, leaves incubated with $1 \mathrm{mM}$ luciferin, and luminescence was recorded with a low-light cooled charge-coupled device camera. For $\mathrm{NaCl}$ treatment, leaf discs incubated with $1 \mathrm{mM}$ luciferin containing $200 \mathrm{mM} \mathrm{NaCl}$ in a 96-well plate for 5-30 min, and the Luc signal was collected by the GLOMAX 96 microplate luminometer.

SOS recruitment system. SRS assays were performed using S. cerevisiae strain JP837, which is a derivative of AXT3K (Dena1:HIS3:ena4, nha1:LEU2, and nhx1: KanMX) as described below. Briefly, the coding sequences of SOS2T168D/ $3308 / \mathrm{S} 294 \mathrm{~A}$ and SOS2 $2^{\mathrm{T} 168 \mathrm{D} / \Delta 308 / \mathrm{S} 294 \mathrm{D}}$ were cloned into $p 416 G P D$ vectors, PKS5, PKS5-3 and PKS5-4 were cloned into $p 414 G P D$ vectors, $14-3-3 \lambda$ and $\omega$ were cloned into p415GPD vectors. The resulting plasmids were transformed into $S$. cerevisiae strain JP837 by a standard lithium-polyethylene glycol method and at least two positive clones were used for further studies. Salt tolerance of yeast were detected on AP medium ( $1 \mathrm{mM} \mathrm{KCl}, 8 \mathrm{mM}$ phosphoric acid, $0.2 \mathrm{mM} \mathrm{CaCl}_{2}, 2 \mathrm{mM} \mathrm{MgSO}_{4}, 10 \mathrm{mM}$ L-Arg, $2 \%$ glucose, plus trace elements, adjust to $\mathrm{pH} 6.5$ with $\mathrm{Arg}$ ) with or without different concentration of $\mathrm{NaCl}$, and grown for $3-4$ days at $28^{\circ} \mathrm{C}$.

In vitro pull-down and in vivo Co-immunoprecipitation assays. For in vitro pull-down assays, all recombinant proteins were purified from E. coli DE3 strains. Briefly, GST-tagged SOS2-JD protein retained on the beads incubated 
with His-tagged PKS5 in kinase buffer with ATP at $30^{\circ} \mathrm{C}$ for $30 \mathrm{~min}$. After removed His-tagged PKS5 by washing three times with PBS buffer, GST-tagged SOS2-JD protein were incubated with His-tagged 14-3-3 $\lambda$ in binding buffer $(2 \mathrm{mM}$ DTT, $10 \mathrm{mM} \mathrm{MgCl}_{2}$ and $20 \mathrm{mM}$ Tris- $\mathrm{HCl}, \mathrm{pH} \mathrm{7.2)}$ at $4{ }^{\circ} \mathrm{C}$ for $3 \mathrm{~h}$, and then the beads were wash for three times with PBS buffer. The pulled-down proteins were detected by immunoblot analysis using an anti-14-3-3 antibody (Santa Cruz SC-33752, 1/2000).

For in vivo Co-immunoprecipitation assays, stable transgenic plants were used to detect the interaction between 14-3-3 proteins and SOS2/PKS5 and the phosphorylation of SCaBP8 $8^{\mathrm{Ser} 237}$ in planta. Total proteins were extracted from Arabidopsis using IP buffer (10 mM Tris, pH 7.5, 2 mM EDTA, $150 \mathrm{mM} \mathrm{NaCl}$, $0.5 \%$ Nonidet P-40, $1 \mathrm{mM}$ phenylmethylsulfonyl fluoride, and $1 \%$ protease inhibitor cocktail; Roche). After removed cellular debris, the supernatant was incubated with anti-C-Myc agarose (Sigma-Aldrich) at $4{ }^{\circ} \mathrm{C}$ for $3 \mathrm{~h}$, and then the beads were washed for five times with IP buffer. The associated proteins were analyzed by immunoblots and detected with anti-C-Myc (CWBIO 01217/10153, 1/ 3000) and anti-P-SC8 (made by AbMart, 1/2000) antibodies.

RT-PCR analysis. Total RNA was extracted from the transgenic $N$. benthamiana plants with Trizol reagent (Invitrogen). RNase-free DNase I (TaKaRa) was used to remove genomic DNA, and the RNA was reverse transcribed using M-MLV reverse transcriptase (Promega). Resulting cDNAs were used to detect the genes expression by RT-PCR analysis. The specific primers were listed in Supplementary Table 1.

Salt and alkali sensitive assays. For salt tolerance assays, 5-day-old seedlings grown on MS medium were transformed to medium containing 75, 100, or 125 $\mathrm{mM} \mathrm{NaCl}$. For alkali tolerance assays, 7-day-old seedlings were transformed to medium at $\mathrm{pH} 6.5$ or 6.7 , which was adjusted by $500 \mathrm{mM} \mathrm{NaHCO}$. All the seedlings were grown in a light growth chamber at $22-24^{\circ} \mathrm{C}$ under a $16-\mathrm{h}$ light and 8-h dark photoperiod.

Plasma membrane $\mathbf{H}^{+}$-ATPase activity assays. The plasma membrane $\mathrm{H}^{+}$ATPase activity assays were performed as described below. Plasma membraneenriched vesicles were isolated from 5-week-old plants treated with $250 \mathrm{mM} \mathrm{NaCl}$ for 3 days. Fifty micrograms of plasma membrane protein was prepared for further measurement. When used, $500 \mathrm{ng}$ of recombinant protein was incubated with plasma membrane protein at room temperature for $10 \mathrm{~min}$. The activity of the $\mathrm{H}^{+}$ATPase was reflected by the change in fluorescence of quinacrine $(\mathrm{a} \mathrm{pH}$-sensitive fluorescent probe), which was obtained by a Hitachi F-7500 imager.

Measurement of $\mathrm{Net} \mathrm{Na}+$ and $\mathrm{H}+$ fluxes with the NMT. The measurement of $\mathrm{Na}^{+}$and $\mathrm{H}^{+}$fluxes was performed as described below. Net flux of $\mathrm{Na}^{+}$and $\mathrm{H}^{+}$ were measured by NMT (YoungerUSA, LLC, Amherst, MA, USA) and imFluxes software. For $\mathrm{Na}^{+}$flux, 7-day-old seedlings were treated in medium with $100 \mathrm{mM}$ $\mathrm{NaCl}$ for $24 \mathrm{~h}$. Pre-pulled and silanized microsensor $(\varnothing 4.5 \pm 0.5 \mu \mathrm{m}, \mathrm{XY}-\mathrm{CGQ}-01)$ were filled with a backfilling solution $\left(\mathrm{Na}^{+}: 250 \mathrm{mM} \mathrm{NaCl} ; \mathrm{H}^{+}: 15 \mathrm{mM} \mathrm{NaCl}+40\right.$ $\mathrm{mM} \mathrm{KH}_{2} \mathrm{PO}_{4}, \mathrm{pH} \mathrm{7.0)}$ to a length of nearly $1.0 \mathrm{~cm}$ and then $50 \mu \mathrm{m}$ columns of selective liquid ion-exchange cocktails (LIXs, $\mathrm{Na}^{+}$: XY-SJ-Na; $\mathrm{H}^{+}$: XY-SJ-H. Younger, USA) were filled from the tip. Prior to the flux measurement, the seedling was incubated in the measuring solution $\left(\mathrm{Na}^{+}: 0.1 \mathrm{mM} \mathrm{CaCl}_{2}, 0.1 \mathrm{mM} \mathrm{KCl}, 0.5\right.$ $\mathrm{mM} \mathrm{NaCl}$, and $0.3 \mathrm{mM} \mathrm{MES}$, adjusted $\mathrm{pH}$ to 6.0 with Tris- $\mathrm{HCl}(\mathrm{pH} 8.8) ; \mathrm{H}^{+}: 0.1$ $\mathrm{mM} \mathrm{CaCl}_{2}, 0.1 \mathrm{mM} \mathrm{KCl}$, and $0.3 \mathrm{mM} \mathrm{MES}$, adjusted $\mathrm{pH}$ to 7.0 with $1 \mathrm{M} \mathrm{KOH}$ ) for $10 \mathrm{~min}$. The microsensor was calibrated with 0.5 or $5 \mathrm{mM} \mathrm{NaCl}$ in calibration liquid $\left(0.1 \mathrm{mM} \mathrm{CaCl}_{2}, 0.1 \mathrm{mM} \mathrm{KCl}, 0.3 \mathrm{mM}\right.$ MES, pH 6.0) for measuring $\mathrm{Na}^{+}$flux; and with pH 5.5 or 6.5 in calibration liquid $\left(0.1 \mathrm{mM} \mathrm{CaCl}_{2}, 0.1 \mathrm{mM} \mathrm{KCl}, 0.3 \mathrm{mM}\right.$ MES) for measuring $\mathrm{H}^{+}$flux. Ion fluxes were measured in the zone about $300 \mu \mathrm{m}$ from the tip. All the measurement results were exported by using the JCal V3.3 (a free MS Excel spreadsheet, youngerusa.com or xbi.org) and the consumables were provided by Xuyue (Beijing).

MST assays. The binding affinities of $14-3-3 \lambda / \kappa$ with calcium ions were detected by Monolith NT.115 (Nanotemper Technologies). All recombinant proteins were purified from $E$. coli DE3 strains, GST-tagged $14-3-3 \lambda / \kappa$ and GST-tagged CDPK6 were labeled in accordance with the manufacturer's procedure. A volume of $100 \mu \mathrm{L}(10 \mu \mathrm{M})$ proteins were exchanged into a labeling buffer and labeled by dye NT-647-NHS at room temperature for $30 \mathrm{~min}$ in the dark. The labeled proteins were incubated with different concentration of calcium ions or ligandins for 10 min, and then loaded into silica capillaries (Polymicro Technologies). The samples were measured by Monolish NT.115 (NanoTemper Technologies) at $25^{\circ} \mathrm{C}, 20 \%$ MST power and $20 \%$ LED power. The results were analyzed by OriginPro 9.0 software and MO. Affinity Analysis software (V2.2.4).

Reporting summary. Further information on experimental design is available in the Nature Research Reporting Summary linked to this article.

\section{Data availability}

All supporting data from this study are available from the article and Supplementary Information files, or from the corresponding author upon request. The source data underlying Figs. 1c, d, 2a, b, e-h, 3b-d, f-h, 4a, c, e, 5a-c, 6b, c, e, f and 7a-f, and Supplementary Figs. 1c, d, $3 b, d, 4 b, c, e, f, 5 a-h$, and $6 e-g$ are provided in the Source Data file.
Received: 2 July 2018 Accepted: 20 February 2019

Published online: 13 March 2019

\section{References}

1. Reddy, A. S. Calcium: silver bullet in signaling. Plant Sci. 160, 381-404 (2001).

2. Snedden, W. A. \& Fromm, H. Calmodulin as a versatile calcium signal transducer in plants. New Phytol. 151, 35-66 (2001).

3. Yan, C. et al. Injury activates $\mathrm{Ca}^{2+} /$ calmodulin-dependent phosphorylation of JAV1-JAZ8-WRKY51 complex for jasmonate biosynthesis. Mol. Cell 70, 136-149 e137 (2018).

4. Cheng, S. H., Willmann, M. R., Chen, H. C. \& Sheen, J. Calcium signaling through protein kinases. The Arabidopsis calcium-dependent protein kinase gene family. Plant Physiol. 129, 469-485 (2002).

5. Harper, J. F. \& Harmon, A. Plants, symbiosis and parasites: a calcium signalling connection. Nat. Rev. Mol. Cell Biol. 6, 555-566 (2005).

6. Tang, R. J. et al. Tonoplast calcium sensors CBL2 and CBL3 control plant growth and ion homeostasis through regulating V-ATPase activity in Arabidopsis. Cell Res. 22, 1650-1665 (2012).

7. Albrecht, V. et al. The calcium sensor CBL1 integrates plant responses to abiotic stresses. Plant J. 36, 457-470 (2003).

8. Batistic, O. \& Kudla, J. Plant calcineurin B-like proteins and their interacting protein kinases. BBA 1793, 985-992 (2009).

9. Luan, S., Kudla, J., Rodriguez-Concepcion, M., Yalovsky, S. \& Gruissem, W. Calmodulins and calcineurin B-like proteins: calcium sensors for specific signal response coupling in plants. Plant Cell 14 Suppl, S389-S400 (2002).

10. Yang, Y. \& Guo, Y. Elucidating the molecular mechanisms mediating plant salt-stress responses. New Phytol. 217, 523-539 (2018).

11. Zhu, J. K. Abiotic stress signaling and responses in plants. Cell 167, 313-324 (2016).

12. Liu, J., Ishitani, M., Halfter, U., Kim, C. S. \& Zhu, J. K. The Arabidopsis thaliana SOS2 gene encodes a protein kinase that is required for salt tolerance. Proc. Natl Acad. Sci. USA 97, 3730-3734 (2000).

13. Liu, J. P. \& Zhu, J. K. A calcium sensor homolog required for plant salt tolerance. Science 280, 1943-1945 (1998).

14. Quan, R. et al. SCABP8/CBL10, a putative calcium sensor, interacts with the protein kinase SOS2 to protect Arabidopsis shoots from salt stress. Plant Cell 19, 1415-1431 (2007).

15. Shi, H., Ishitani, M., Kim, C. \& Zhu, J. K. The Arabidopsis thaliana salt tolerance gene $\mathrm{SOS} 1$ encodes a putative $\mathrm{Na}^{+} / \mathrm{H}^{+}$antiporter. Proc. Natl Acad. Sci. USA 97, 6896-6901 (2000).

16. Halfter, U., Ishitani, M. \& Zhu, J. K. The Arabidopsis SOS2 protein kinase physically interacts with and is activated by the calcium-binding protein SOS3. Proc. Natl Acad. Sci. USA 97, 3735-3740 (2000).

17. Lin, H. et al. Phosphorylation of SOS3-LIKE CALCIUM BINDING PROTEIN8 by SOS2 protein kinase stabilizes their protein complex and regulates salt tolerance in Arabidopsis. Plant Cell 21, 1607-1619 (2009).

18. Guo, Y., Halfter, U., Ishitani, M. \& Zhu, J. K. Molecular characterization of functional domains in the protein kinase SOS2 that is required for plant salt tolerance. Plant Cell 13, 1383-1399 (2001).

19. Quintero, F. J. et al. Activation of the plasma membrane $\mathrm{Na} / \mathrm{H}$ antiporter SaltOverly-Sensitive 1 (SOS1) by phosphorylation of an auto-inhibitory Cterminal domain. Proc. Natl Acad. Sci. USA 108, 2611-2616 (2011).

20. Quintero, F. J., Ohta, M., Shi, H., Zhu, J. K. \& Pardo, J. M. Reconstitution in yeast of the Arabidopsis SOS signaling pathway for $\mathrm{Na}+$ homeostasis. Proc. Natl Acad. Sci. USA 99, 9061-9066 (2002).

21. Zhou, H. et al. Inhibition of the Arabidopsis salt overly sensitive pathway by 14-3-3 proteins. Plant Cell 26, 1166-1182 (2014).

22. Kim, W. Y. et al. Release of SOS2 kinase from sequestration with GIGANTEA determines salt tolerance in Arabidopsis. Nat. Commun. 4, 1352 (2013).

23. Haruta, M. \& Sussman, M. R. The effect of a genetically reduced plasma membrane protonmotive force on vegetative growth of Arabidopsis. Plant Physiol. 158, 1158-1171 (2012).

24. Fuglsang, A. T. et al. Arabidopsis protein kinase PKS5 inhibits the plasma membrane $\mathrm{H}^{+}$-ATPase by preventing interaction with 14-3-3 protein. Plant Cell 19, 1617-1634 (2007).

25. Fuglsang, A. T. et al. Receptor kinase-mediated control of primary active proton pumping at the plasma membrane. Plant J. 80, 951-964 (2014).

26. Hayashi, Y., Takahashi, K., Inoue, S. \& Kinoshita, T. Abscisic acid suppresses hypocotyl elongation by dephosphorylating plasma membrane $\mathrm{H}^{+}$-ATPase in Arabidopsis thaliana. Plant Cell Physiol. 55, 845-853 (2014).

27. Planes, M. D. et al. A mechanism of growth inhibition by abscisic acid in germinating seeds of Arabidopsis thaliana based on inhibition of plasma membrane $\mathrm{H}^{+}$-ATPase and decreased cytosolic $\mathrm{pH}, \mathrm{K}^{+}$, and anions. J. Exp. Bot. 66, 813-825 (2015). 
28. Rudashevskaya, E. L., Ye, J., Jensen, O. N., Fuglsang, A. T. \& Palmgren, M. G. Phosphosite mapping of P-type plasma membrane $\mathrm{H}^{+}$-ATPase in homologous and heterologous environments. J. Biol. Chem. 287, 4904-4913 (2012).

29. Yang, Y. et al. The Arabidopsis chaperone J3 regulates the plasma membrane $\mathrm{H}^{+}$-ATPase through interaction with the PKS5 kinase. Plant Cell 22, 1313-1332 (2010).

30. Fuglsang, A. T. et al. Binding of 14-3-3 protein to the plasma membrane $\mathrm{H}$ +-ATPase AHA2 involves the three C-terminal residues $\mathrm{Tyr}^{946}$-Thr-Val and requires phosphorylation of $\mathrm{Thr}^{947}$. J. Biol. Chem. 274, 36774-36780 (1999).

31. Day, I. S., Reddy, V. S., Shad Ali, G. \& Reddy, A. S. Analysis of EF-handcontaining proteins in Arabidopsis. Genome Biol. 3, RESEARCH0056 (2002).

32. Lu, G., Sehnke, P. C. \& Ferl, R. J. Phosphorylation and calcium binding properties of an Arabidopsis GF14 brain protein homolog. Plant Cell 6, 501-510 (1994).

33. Lumba, S. et al. A mesoscale abscisic acid hormone interactome reveals a dynamic signaling landscape in Arabidopsis. Dev. Cell 29, 360-372 (2014).

34. Liu, Z. et al. Plasma membrane CRPK1-mediated phosphorylation of 14-3-3 proteins induces their nuclear import to fine-tune CBF signaling during cold response. Mol. Cell 66, 117-128 e115 (2017).

35. Bai, M. Y. et al. Functions of OsBZR1 and 14-3-3 proteins in brassinosteroid signaling in rice. Proc. Natl Acad. Sci. USA 104, 13839-13844 (2007).

36. Ghorbel, M. et al. Regulation of the wheat MAP kinase phosphatase 1 by 14-33 proteins. Plant Sci. 257, 37-47 (2017).

37. Camoni, L., Iori, V., Marra, M. \& Aducci, P. Phosphorylation-dependent interaction between plant plasma membrane $\mathrm{H}^{+}$-ATPase and 14-3-3 proteins. J. Biol. Chem. 275, 9919-9923 (2000).

38. Maudoux, $\mathrm{O}$. et al. A plant plasma membrane $\mathrm{H}^{+}$-ATPase expressed in yeast is activated by phosphorylation at its penultimate residue and binding of 14-3-3 regulatory proteins in the absence of fusicoccin. J. Biol. Chem. 275, $17762-17770$ (2000).

\section{Acknowledgements}

This work was supported by the National Basic Research Program of China (2015CB910202) and the National Natural Science Foundation of China (31430012, 31670260, 31861133005, U1706201)

\section{Author contributions}

Y.G., Y.Y. and Z.Y. conceived and designed the research plans; Z.Y., C.W., Y.X. and X.L. performed the experiments; Y.G., Z.Y., C.S. and Y.Y. analyzed data. S.C. generated and analyzed MS/MS data; Y.G., Z.Y., C.S. and Y.Y. wrote the paper.

\section{Additional information}

Supplementary Information accompanies this paper at https://doi.org/10.1038/s41467019-09181-2.

Competing interests: The authors declare no competing interests.

Reprints and permission information is available online at http://npg.nature.com/ reprintsandpermissions/

Journal peer review information: Nature Communications thanks the anonymous reviewers for their contribution to the peer review of this work. Peer reviewer reports are available.

Publisher's note: Springer Nature remains neutral with regard to jurisdictional claims in published maps and institutional affiliations.

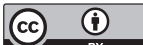

Open Access This article is licensed under a Creative Commons Attribution 4.0 International License, which permits use, sharing, adaptation, distribution and reproduction in any medium or format, as long as you give appropriate credit to the original author(s) and the source, provide a link to the Creative Commons license, and indicate if changes were made. The images or other third party material in this article are included in the article's Creative Commons license, unless indicated otherwise in a credit line to the material. If material is not included in the article's Creative Commons license and your intended use is not permitted by statutory regulation or exceeds the permitted use, you will need to obtain permission directly from the copyright holder. To view a copy of this license, visit http://creativecommons.org/ licenses/by/4.0/.

(C) The Author(s) 2019 